\title{
MODERATE DEVIATIONS AND NONPARAMETRIC INFERENCE FOR MONOTONE FUNCTIONS
}

\author{
By FuQING GAO ${ }^{1}$, JiE XIONG ${ }^{2}$ AND XINGQIU ZHAO ${ }^{3}$ \\ Wuhan University, University of Macau and \\ The Hong Kong Polytechnic University
}

\begin{abstract}
This paper considers self-normalized limits and moderate deviations of nonparametric maximum likelihood estimators for monotone functions. We obtain their self-normalized Cramér-type moderate deviations and limit distribution theorems for the nonparametric maximum likelihood estimator in the current status model and the Grenander-type estimator. As applications of the results, we present a new procedure to construct asymptotical confidence intervals and asymptotical rejection regions of hypothesis testing for monotone functions. The theoretical results can guarantee that the new test has the probability of type II error tending to 0 exponentially. Simulation studies also show that the new nonparametric test works well for the most commonly used parametric survival functions such as exponential and Weibull survival distributions.
\end{abstract}

1. Introduction. The maximum likelihood estimator (MLE) of a monotone density function is the Grenander estimator [see Grenander (1956), Prakasa Rao (1969), and Groeneboom (1985)]. The asymptotic distribution of the Grenander estimator was obtained in Prakasa Rao (1969). Groeneboom and Wellner (1992) derived the limit distribution of the nonparametric maximum likelihood estimator (NPMLE) for current status data. Banerjee and Wellner (2001) proposed a likelihood ratio (LR) test for monotone functions to construct pointwise confidence intervals for the monotone functions and obtained its limit distribution. The method was studied further in Banerjee and Wellner (2005a, 2005b) and Banerjee (2007). Banerjee and Wellner (2001, 2005a, 2005b) investigated the power behavior of the LR test, and demonstrated the superior performance of the LR test over several existing methods in the current status model. Recently,

Received September 2016; revised March 2017.

${ }^{1}$ Supported by the National Natural Science Foundation of China $(11171262,11571262)$, and the Specialized Research Fund for the Doctoral Program of Higher Education (SRFDP) of China Grant 20130141110076.

${ }^{2}$ Supported by Macao Science and Technology Fund FDCT 076/2012/A3 and Multi-Year Research Grants of the University of Macau No. MYRG2014-00015-FST and MYRG2014-00034-FST.

${ }^{3}$ Supported by the Research Grant Council of Hong Kong (503513), the National Natural Science Foundation of China (No. 1371299) and The Hong Kong Polytechnic University.

MSC2010 subject classifications. Primary 60F10, 62G20; secondary 62G07.

Key words and phrases. Grenander estimator, interval censored data, large deviations, moderate deviations, nonparametric MLE, self-normalized limit, strong approximation, Talagrand inequality. 
Groeneboom, Jongbloed and Witte (2010) proposed two estimators: the maximum smoothed likelihood and the smoothed maximum likelihood estimators and established their asymptotic normality, but the asymptotic variances depend on the derivative of the density function. Moreover, Groeneboom (2014) studied the maximum smoothed likelihood estimator for the interval censoring model, Groeneboom and Jongbloed (2014) discussed these nonparametric estimators, and Groeneboom and Jongbloed (2015) provided an alternative way to deduce the distribution of the likelihood ratio and extended it to the Grenander estimator.

Asymptotic behavior for Grenander's estimator and its extensions have been studied widely in the last 30 years. The asymptotic normality of the $L_{1}$-distance between the decreasing density and its Grenander estimator was obtained by Groeneboom, Hooghiemstra and Lopuhaä (1999), and a similar result for monotone regression can be found in Durot (2002). Huang and Wellner (1995) studied the limit distribution of a Grenander-type estimator for a monotone hazard rate in a right-censoring model. Durot (2007) gave the limit distribution for the $L_{p}$-error of a Grenander-type estimator. Recently, the limiting distribution of the supremum distance was studied by Durot, Kulikov and Lopuhaä (2012), and a law of the iterated logarithm for the Grenander's estimator was established by Dümbgen, Wellner and Wolff (2016).

Recently, self-normalized Cramér-type moderate deviations in statistics have attracted wide attention; see, for example, Liu and Shao (2010, 2013), Chang, Shao and Zhou (2016). In these papers, the statistics can be represented analytically by samples. The NPMLE of current status data and Grenander-type estimator are inverse statistical problems, and their weak limits are the maximum point of a Brownian motion minus a parabola. In this paper, we study self-normalized limit distributions and Cramér-type moderate deviations of the NPMLE for monotone functions. The self-normalized method is based on the self-normalized Cramér-type moderate deviations, and the self-normalized limit distributions for the NPMLE in the current status model and Grenander-type estimator are obtained. As applications, we use these results to construct asymptotical confidence intervals and asymptotical rejection regions of hypothesis testing for monotone functions. The new procedure for statistical inference is different from the LR method in Banerjee and Wellner (2001) and Groeneboom and Jongbloed (2015) and the smoothed maximum likelihood method in Groeneboom, Jongbloed and Witte (2010). Theoretically, the proposed test has the probability of type II error tending to 0 with exponential decay by moderate deviations. Such theoretical properties of the type 2 error are not generally well studied for other competing methods.

The rest of the paper is organized as follows. Section 2 presents the main results including the self-normalized Cramér-type moderate deviations, and the selfnormalized limit distributions for the NPMLE in the current status model and Grenander-type estimator. In Section 3, the main results in the current status model are proved using a general Cramér-type moderate deviation theorem and a general 
limit distribution theorem. The proofs of these general results are postponed to Sections 5. Our proofs are based on the strong approximation, Talagrand deviation inequality and small ball estimates. In Section 4, we use the theoretical results to construct statistical hypothesis tests and pointwise confidence intervals. In addition, we conduct simulation studies to evaluate the finite-sample performance of the proposed method and compare it with the LR test in Section 4. The proofs of some technical lemmas and the results for Grenander-type estimator are given in Appendix A of the Supplementary Material [Gao, Xiong and Zhao (2017)], and some useful known results are presented in Appendix B of the Supplementary Material [Gao, Xiong and Zhao (2017)] for the convenience of the reader.

2. Main results. We consider two types of problems: the NPMLE in the current status model and the Grenander-type estimator.

2.1. Current status data. Consider a study that concerns the time to occurrence of a certain event of interest such as failure or onset of a disease. In many cases, the event time cannot be observed completely since it is often too expensive or even impossible to observe the exact event occurrence time. However, the current status of the subjects at a random examination time can be much more easily observed. This means that for each subject, the event occurrence time is unknown, but it is known whether the event has occurred before the examination time. Such data are called current status data or case 1 interval censored data in survival analysis [Groeneboom and Wellner (1992), Huang and Wellner (1995), Groeneboom (1996), Sun (2006), Groeneboom, Jongbloed and Witte (2010)].

Let $X_{1}, \ldots, X_{n}$ and $T_{1}, \ldots, T_{n}$ be independent i.i.d. samples from unknown distribution functions $F_{0}$ and $G$ on the nonnegative half-line, respectively. Set $\delta_{i}=$ $I_{\left\{X_{i} \leq T_{i}\right\}}$. Here, $X_{i}$ is interpreted as the (unobserved) time of onset of a disease; $T_{i}$ is a check-up time at which patient $i$ is observed to be ill or not: $\delta_{i}=1$ or 0 . The observations consist of the pairs $\left(\delta_{1}, T_{1}\right), \ldots,\left(\delta_{n}, T_{n}\right)$ in the current status model. The NPMLE $\hat{F}_{n}$ for $F_{0}$ is defined to be the one that maximizes the log-likelihood function

$$
F \mapsto \sum_{i=1}^{n}\left(\delta_{i} \log F\left(T_{i}\right)+\left(1-\delta_{i}\right) \log \left(1-F\left(T_{i}\right)\right)\right),
$$

where $F$ is a right-continuous distribution function. Since the likelihood function depends on $F$ only through the values $F\left(T_{i}\right)$, the NPMLE is not unique. To avoid this ambiguity, $\hat{F}_{n}$ is assumed to be a constant on each interval $\left[T_{(i-1)}, T_{(i)}\right)$, where $T_{(i)}$ is the $i$ th order statistic of $T_{1}, \ldots, T_{n}$. A basic assumption is as follows.

Assumption (A1). Let $t_{0}>0$ be a fixed point such that $0<F_{0}\left(t_{0}\right)$, $G\left(t_{0}\right)<1$. Let $F_{0}$ and $G$ be continuous on $[0, \infty)$ and differentiable on a closed neighborhood $\left[t_{0}-\epsilon_{0}, t_{0}+\epsilon_{0}\right]$ of the point $t_{0}$, with strictly positive derivatives $f_{0}(t)$ and $g(t), t \in\left[t_{0}-\epsilon_{0}, t_{0}+\epsilon_{0}\right]$, respectively, where $0<\epsilon_{0}<t_{0}$. 
Throughout the paper, the notation $\xi_{n} \stackrel{d}{\rightarrow} \xi$ means that the random sequence $\xi_{n}$ converges to a random variable $\xi$ in distribution. The notation $\operatorname{argmin}_{t \in U} f(t)$ [resp., $\left.\operatorname{argmax}_{t \in U} f(t)\right]$ stands for the point $t \in U$ at which $f(t)$ is minimized (resp., maximized), where the largest value is chosen when multiple minimizers (resp., maximizers) exist.

Groeneboom and Wellner (1992) established the strong consistency of the NPMLE, and derived the limit distribution of the NPMLE $\hat{F}_{n}\left(t_{0}\right)$ for a fixed point $t_{0}$ as follows: under Assumption (A1) stated above,

$$
\frac{n^{1 / 3}\left(\hat{F}_{n}\left(t_{0}\right)-F_{0}\left(t_{0}\right)\right)}{\left(\sigma_{F}^{2}\left(t_{0}\right)\right)^{1 / 3}} \stackrel{d}{\rightarrow} \tau
$$

where

$$
\sigma_{F}^{2}\left(t_{0}\right):=\frac{4 F_{0}\left(t_{0}\right)\left(1-F_{0}\left(t_{0}\right)\right) f_{0}\left(t_{0}\right)}{g\left(t_{0}\right)}, \quad \tau:=\underset{t \in \mathbb{R}}{\operatorname{argmin}}\left\{W(t)+t^{2}\right\},
$$

and $\{W(t), t \in \mathbb{R}\}$ is a two-sided Wiener process with $W(0)=0$.

By Corollaries 3.3 and 3.4 in Groeneboom (1989), the density function $f_{\tau}$ of $\tau$ is symmetric and bounded continuous, and has the tail behavior

$$
f_{\tau}(x) \sim \frac{2}{\mathrm{Ai}^{\prime}\left(a_{1}\right)} 4^{1 / 3}|x| \exp \left\{-\frac{2}{3}|x|^{3}+2^{1 / 3} a_{1}|x|\right\}, \quad|x| \rightarrow \infty
$$

where $a_{1} \approx-2.3381$ is the largest zero of the Airy function $\mathrm{Ai}$ and $\operatorname{Ai}^{\prime}\left(a_{1}\right) \approx$ 0.7002. By Lemma 2.1 in Hooghiemstra and Lopuhaä (1998), the distribution function $F_{\tau}$ of $\tau$ satisfies

$$
1-F_{\tau}(x) \sim \frac{1}{\mathrm{Ai}^{\prime}\left(a_{1}\right)} 4^{1 / 3} \frac{1}{x} \exp \left\{-\frac{2}{3} x^{3}+2^{1 / 3} a_{1} x\right\}, \quad x \rightarrow+\infty .
$$

In order to use Groeneboom and Wellner's (1992) result for statistical inference, we need to construct a consistent estimator of $f_{0}$ or to obtain a self-normalized limit distribution of the NPMLE. This is one of our motivations. Another motivation of the paper is to study the tail probabilities of $\hat{F}_{n}\left(t_{0}\right)-F_{0}\left(t_{0}\right)$ and to construct confidence intervals and rejection regions for hypothesis testing for $F_{0}\left(t_{0}\right)$. We consider the following asymptotic behavior of $\hat{F}_{n}\left(t_{0}\right)-F_{0}\left(t_{0}\right)$ : Cramér-type moderate deviation and moderate deviation principle (MDP).

The Cramér-type moderate deviation is to find $b_{n} \rightarrow \infty$ such that

$$
\sup _{x \in\left[0, b_{n}\right]}\left|\frac{1}{1-F_{\tau}(x)} P\left(\frac{n^{1 / 3}\left(\hat{F}_{n}\left(t_{0}\right)-F_{0}\left(t_{0}\right)\right)}{\left(\sigma_{F}^{2}\left(t_{0}\right)\right)^{1 / 3}} \geq x\right)-1\right| \rightarrow 0 .
$$

The moderate deviation principle (MDP) is to find two suitable sequences of positive numbers $b_{n} \rightarrow \infty$ and $\beta_{n} \rightarrow \infty$ with $\frac{n^{1 / 3}}{b_{n}} \rightarrow \infty$ such that

$$
P\left(\left|\frac{1}{b_{n}} \frac{n^{1 / 3}\left(\hat{F}_{n}\left(t_{0}\right)-F_{0}\left(t_{0}\right)\right)}{\left(\sigma_{F}^{2}\left(t_{0}\right)\right)^{1 / 3}}\right|>x\right) \approx e^{-I(x) \beta_{n}},
$$


where $I(x)$ is a suitable rate function and $\beta_{n}$ is called the speed. If one replaces $\frac{n^{1 / 3}}{b_{n}} \rightarrow \infty$ by $\frac{n^{1 / 3}}{b_{n}} \rightarrow c>0$, this result is called the large deviation principle (LDP). Generally, the rate function $I$ of the MDP can be easily calculated, and provides a useful method for statistical inference. Some notion and properties in large deviation are given in Appendix B of the Supplementary Material [Gao, Xiong and Zhao (2017)].

Generally, the bigger $b_{n}$ is, the faster the convergence rate is. In the i.i.d. case, $b_{n}=O\left(n^{1 / 6}\right)$ is the best for the Cramér-type moderate deviation [see Petrov (1975), de la Peña, Lai and Shao (2009)]. For the NPMLE, due to a technical reason, we assume that $b_{n}$ satisfies, as $n \rightarrow \infty$,

$$
b_{n} \rightarrow \infty \text { and } \frac{b_{n}^{10} \log n}{n^{1 / 3}} \rightarrow 0 .
$$

That is, $b_{n} \rightarrow \infty$ and $b_{n}=o\left(n^{1 / 30} /(\log n)^{1 / 10}\right)$. tion.

To study moderate deviations of the NPMLE, we need the following assump-

Assumption (A2). The functions $f_{0}(t)$ and $g(t)$ are Lipschitz continuous on $\left[t_{0}-\epsilon_{0}, t_{0}+\epsilon_{0}\right]$.

Let $\hat{g}_{n}$ be a classical kernel density estimator of $g_{0}$ defined by

$$
\hat{g}_{n}(t)=\frac{1}{n h_{n}} \sum_{k=1}^{n} I_{[0,1]}\left(\frac{t-T_{k}}{h_{n}}\right),
$$

where $h_{n} \rightarrow 0$ and $\frac{n h_{n}}{b_{n}^{3}} \rightarrow \infty$. Then by the large deviation principle for the classical kernel density estimator [see the proof of Proposition 3.1 in Gao (2003)], for any $r>0$,

$$
\lim _{n \rightarrow \infty} \frac{1}{b_{n}^{3}} \log P\left(\left|\hat{g}_{n}\left(t_{0}\right)-g_{0}\left(t_{0}\right)\right|>r\right)=-\infty,
$$

since $\lim _{n \rightarrow \infty} \frac{n h_{n}}{b_{n}^{3}}=\infty$. In order to obtain a faster speed, one can take $h_{n}=\frac{1}{\log n}$. We can also replace $\log n$ by a sequence $r_{n}$ with $\log n \leq r_{n}=o\left(n^{1 / 3} / b_{n}\right)$ to obtain a faster convergence speed.

Let us introduce two estimators of $f_{0}$ as follows:

$$
\hat{f}_{n}(t)=\frac{n^{1 / 3}}{b_{n} \log n}\left(\hat{F}_{n}\left(t+b_{n} \log n / n^{1 / 3}\right)-\hat{F}_{n}(t)\right)
$$

and

$$
\tilde{f}_{n}(t)=\frac{n^{1 / 3}}{\log n}\left(\hat{F}_{n}\left(t+\log n / n^{1 / 3}\right)-\hat{F}_{n}(t)\right) .
$$

For the estimators $\hat{f}_{n}(t)$ and $\tilde{f}_{n}(t)$, we also have the following exponential convergence. 
Proposition 2.1. (i) Assume that Assumptions (A1) and (A2) hold. Then for any $r>0$,

$$
\lim _{n \rightarrow \infty} \frac{1}{b_{n}^{3}} \log P\left(\left|\hat{F}_{n}\left(t_{0}\right)-F_{0}\left(t_{0}\right)\right|>r\right)=-\infty
$$

and

$$
\lim _{n \rightarrow \infty} \frac{1}{b_{n}^{3}} \log P\left(\left|\hat{f}_{n}\left(t_{0}\right)-f_{0}\left(t_{0}\right)\right|>r\right)=-\infty .
$$

(ii) Assume that Assumption (A1) holds. Then for any $r>0$,

$$
\lim _{n \rightarrow \infty} P\left(\left|\tilde{f}_{n}\left(t_{0}\right)-f_{0}\left(t_{0}\right)\right|>r\right)=0
$$

Now, we state the self-normalized Cramér-type moderate deviations and the self-normalized limit distribution theorem for the NPMLE in the current status model.

THEOREM 2.1. Assume that Assumptions (A1) and (A2) hold. Then for each $\rho>0$, uniformly for $0 \leq x \leq \rho b_{n}$,

$$
\frac{1}{1-F_{\tau}(x)} P\left( \pm \frac{n^{1 / 3}\left(\hat{F}_{n}\left(t_{0}\right)-F_{0}\left(t_{0}\right)\right)}{\left(\hat{\sigma}_{n}^{2}\left(t_{0}\right)\right)^{1 / 3}} \geq x\right) \rightarrow 1
$$

that is,

$$
\frac{1}{1-F_{\tau}(x)} P\left(\frac{n^{1 / 3}\left(\hat{F}_{n}\left(t_{0}\right)-F_{0}\left(t_{0}\right)\right)}{\left(\hat{\sigma}_{n}^{2}\left(t_{0}\right)\right)^{1 / 3}} \geq x\right) \rightarrow 1
$$

and

$$
\frac{1}{1-F_{\tau}(x)} P\left(\frac{n^{1 / 3}\left(\hat{F}_{n}\left(t_{0}\right)-F_{0}\left(t_{0}\right)\right)}{\left(\hat{\sigma}_{n}^{2}\left(t_{0}\right)\right)^{1 / 3}} \leq-x\right) \rightarrow 1,
$$

where $\hat{\sigma}_{n}^{2}\left(t_{0}\right):=4 \hat{F}_{n}\left(t_{0}\right)\left(1-\hat{F}_{n}\left(t_{0}\right)\right) \hat{f}_{n}\left(t_{0}\right) / \hat{g}_{n}\left(t_{0}\right)$.

In particular, the sequence $\left\{\frac{n^{1 / 3}}{b_{n}} \frac{\hat{F}_{n}\left(t_{0}\right)-F_{0}\left(t_{0}\right)}{\left(\hat{\sigma}_{n}^{2}\left(t_{0}\right)\right)^{1 / 3}}, n \geq 1\right\}$ satisfies the MDP in $\mathbb{R}$ with speed $b_{n}^{3}$ and rate function $I(x)=\frac{2}{3}|x|^{3}$, that is, for any open subset $A$ of $\mathbb{R}$,

$$
\liminf _{n \rightarrow \infty} \frac{1}{b_{n}^{3}} \log P\left(\frac{n^{1 / 3}}{b_{n}} \frac{\hat{F}_{n}\left(t_{0}\right)-F_{0}\left(t_{0}\right)}{\left(\hat{\sigma}_{n}^{2}\left(t_{0}\right)\right)^{1 / 3}} \in A\right) \geq-\inf _{x \in A} I(x),
$$

and for any closed subset $B$ of $\mathbb{R}$,

$$
\limsup _{n \rightarrow \infty} \frac{1}{b_{n}^{3}} \log P\left(\frac{n^{1 / 3}}{b_{n}} \frac{\hat{F}_{n}\left(t_{0}\right)-F_{0}\left(t_{0}\right)}{\left(\hat{\sigma}_{n}^{2}\left(t_{0}\right)\right)^{1 / 3}} \in B\right) \leq-\inf _{x \in B} I(x) .
$$


THEOREM 2.2. Assume that Assumption (A1) holds. Then

$$
\frac{n^{1 / 3}\left(\hat{F}_{n}\left(t_{0}\right)-F_{0}\left(t_{0}\right)\right)}{\left(\tilde{\sigma}_{n}^{2}\left(t_{0}\right)\right)^{1 / 3}} \stackrel{d}{\rightarrow} \tau,
$$

where $\tilde{\sigma}_{n}^{2}\left(t_{0}\right):=4 \hat{F}_{n}\left(t_{0}\right)\left(1-\hat{F}_{n}\left(t_{0}\right)\right) \tilde{f}_{n}\left(t_{0}\right) / \hat{g}_{n}\left(t_{0}\right)$.

2.2. Grenander-type estimator. Let $\lambda$ be a decreasing (or increasing) function on $[0,1]$ and $\Lambda(t)=\int_{0}^{t} \lambda(u) d u, t \in[0,1]$. Let $\Lambda_{n}(t)$ be a cadlag (right continuous with finite left-hand limits at every point) step process and let $\hat{\Lambda}_{n}$ be the least concave majorant (resp., greatest convex majorant) of $\Lambda_{n}$. The Grenander-type estimator $\hat{\lambda}_{n}$ of $\lambda$ is defined as the left derivative of $\hat{\Lambda}_{n}$. Then $\hat{\lambda}_{n}$ is monotone, left-continuous and piecewise constant.

We introduce the following assumptions.

(GA1) The function $\lambda$ is monotone and differentiable on $[0,1]$,

$$
\inf _{t \in[0,1]}\left|\lambda^{\prime}(t)\right|>0 \quad \text { and } \sup _{t \in[0,1]}\left|\lambda^{\prime}(t)\right|<\infty .
$$

(GA2) Let $W$ be either a Brownian bridge or a Brownian motion. There exist positive absolute constants $C, K, \kappa, L:[0,1] \rightarrow \mathbb{R}$ and versions of $\Lambda_{n}$ and $W$ such that for all $r \in[0, n]$,

$$
\begin{aligned}
& P\left(\sup _{u \in[0,1]}\left|n\left(\Lambda_{n}(u)-\Lambda(u)\right)-n^{1 / 2} W(L(u))\right| \geq C \log n+r\right) \\
& \quad \leq K \exp \{-\kappa r\} .
\end{aligned}
$$

Moreover, $L$ is increasing and twice differentiable in $(0,1)$, and $L^{\prime}(s)>0$ for any $s \in(0,1)$.

We define an estimator of $\lambda^{\prime}$ as follows:

$$
\hat{\lambda}_{n}^{(1)}(t)=\frac{n^{1 / 3}}{b_{n} \log n}\left(\hat{\lambda}_{n}\left(t+b_{n} \log n / n^{1 / 3}\right)-\hat{\lambda}_{n}(t)\right) .
$$

The estimators $\hat{\lambda}_{n}(t)$ and $\hat{\lambda}_{n}^{(1)}(t)$ also have exponential convergence.

Proposition 2.2. Assume that (GA1) and (GA2) hold.

(i) For any $r>0$, we have

(2.17) $\lim _{n \rightarrow \infty} \frac{1}{b_{n}^{3}} \log \max \left\{P\left(\left|\hat{\lambda}_{n}(t)-\lambda(t)\right|>r\right), P\left(\left|\hat{\lambda}_{n}^{(1)}(t)-\lambda^{\prime}(t)\right|>r\right)\right\}=-\infty$.

(ii) If $\liminf _{n \rightarrow \infty} \frac{b_{n}}{(\log n)^{3}}>1$, then

$$
P\left(\lim _{n \rightarrow \infty} \hat{\lambda}_{n}(t)=\lambda(t)\right)=1 \quad \text { and } \quad P\left(\lim _{n \rightarrow \infty} \hat{\lambda}_{n}^{(1)}(t)=\lambda^{\prime}(t)\right)=1 .
$$


The following result is the self-normalized Cramér-type moderate deviations for the Grenander-type estimator.

THEOREM 2.3. Assume that (GA1) and (GA2) hold. Then for $t \in(0,1)$ and $\rho>0$, uniformly for $0 \leq x \leq \rho b_{n}$,

$$
\frac{1}{1-F_{\tau}(x)} P\left( \pm \frac{n^{1 / 3}\left(\hat{\lambda}_{n}(t)-\lambda(t)\right)}{\left(4 L^{\prime}(t)\left|\hat{\lambda}_{n}^{(1)}(t)\right|\right)^{1 / 3}} \geq x\right) \rightarrow 1 .
$$

In particular, the sequence $\left\{\frac{n^{1 / 3}}{b_{n}} \frac{\hat{\lambda}_{n}(t)-\lambda(t)}{\left(4 L^{\prime}(t)\left|\hat{\lambda}_{n}^{(1)}(t)\right|\right)^{1 / 3}}, n \geq 1\right\}$ satisfies the MDP in $\mathbb{R}$ with speed $b_{n}^{3}$ and rate function $I(x)=\frac{2}{3}|x|^{3}$.

The next subsections give several corollaries of Theorem 2.3.

2.2.1. The monotone density function. Let $X_{1}, \ldots, X_{n}$ be independent random variables taking values in $[0,1]$ with the common distribution function $F$ and the monotone density function $f$. Then the monotone estimator based on the empirical distribution function of $X_{1}, \ldots, X_{n}$ is the Grenander estimator $\hat{f}_{n}$.

COROllary 2.1. Assume that (GA1) holds for $f$. Then for $t \in(0,1)$ and $\rho>0$, uniformly for $0 \leq x \leq \rho b_{n}$,

$$
\frac{1}{1-F_{\tau}(x)} P\left( \pm \frac{n^{1 / 3}\left(\hat{f}_{n}(t)-f(t)\right)}{\left(4 \hat{f}_{n}(t)\left|\hat{f}_{n}^{(1)}(t)\right|\right)^{1 / 3}} \geq x\right) \rightarrow 1 .
$$

2.2.2. Monotone hazard function with right-censored data. Let $\left(X_{1}, \delta_{1}\right), \ldots$, $\left(X_{n}, \delta_{n}\right)$ be a right-censored sample, where $X_{i}=\min \left\{T_{i}, Y_{i}\right\}, \delta_{i}=I_{\left\{T_{i} \leq Y_{i}\right\}}, T_{i}$, $i=1, \ldots, n$ are nonnegative i.i.d. failure times, and $Y_{i}, i=1, \ldots, n$ are i.i.d. censoring times independent of $T_{i}, i=1, \ldots, n$. Assume that the common distribution function $F$ of $T_{i}, i=1, \ldots, n$ is absolutely continuous with density function $f$. Assume that $F$ has a monotone hazard rate $\lambda=f /(1-F)$. Let $N_{n}$ be the Nelson-Aalen estimator, defined as follows: if $t_{1}<\cdots<t_{k}$ are the distinct uncensored times and $n_{j}$ is the number of $X_{i}$ 's that are greater than or equal to $t_{j}$ $(j=1, \ldots, k)$, then

$$
N_{n}(t)=\sum_{i=1}^{k-1} I_{\left[t_{i}, t_{i+1}\right)}(t) \sum_{j \leq i} \frac{1}{n_{j}}+I_{\left[t_{k}, 1\right]}(t) \sum_{j \leq k} \frac{1}{n_{j}} .
$$

Let $\Lambda_{n}$ be the restriction of $N_{n}$ to $[0,1]$ and let $G$ be the common distribution function of $Y_{i}, i=1, \ldots, n$. Let $\hat{\lambda}_{n}$ be the monotone estimator based on $\Lambda_{n}$ [see Huang and Wellner (1995)]. Let $F_{n}$ an $G_{n}$ be the Kaplan-Meier estimators of $F$ and $G$, respectively. 
Corollary 2.2. Suppose that (GA1) holds, $F(1)<1, \lim _{t \rightarrow 1} G(t)<1$, and $G$ has a bounded continuous first derivative on $(0,1)$. Then for $t \in(0,1)$ and $\rho>0$, uniformly for $0 \leq x \leq \rho b_{n}$,

$$
\frac{1}{1-F_{\tau}(x)} P\left( \pm \frac{n^{1 / 3}\left(\hat{\lambda}_{n}(t)-\lambda(t)\right)\left(1-F_{n}(t)\right)\left(1-G_{n}(t)\right)}{\left(4 \hat{\lambda}_{n}(t)\left|\hat{\lambda}_{n}^{(1)}(t)\right|\right)^{1 / 3}} \geq x\right) \rightarrow 1 .
$$

2.2.3. The monotone regression model. Consider the model

$$
Y_{i, n}=\lambda(i / n)+\varepsilon_{i, n}, \quad i=1, \ldots, n,
$$

where $\varepsilon_{i, n}, i=1, \ldots, n$, are independent random variables with mean zero. Let

$$
\Lambda_{n}(t)=\frac{1}{n} \sum_{i \leq n t} Y_{i, n}, \quad t \in[0,1] .
$$

Then the monotone estimator $\hat{\lambda}_{n}$ based on $\Lambda_{n}$ is a slight modification of the Brunk estimator.

COROLlARY 2.3. Suppose that (GA1) holds and there exists $\delta>0$ such that $\sup _{i, n} E\left(\exp \left\{\delta\left|\varepsilon_{i, n}\right|\right\}\right)<\infty$. Assume, moreover, that $\operatorname{var}\left(\varepsilon_{i, n}\right)=\sigma^{2}(i / n)$ for some $\sigma^{2}:[0,1] \rightarrow \mathbb{R}_{+}$. If $\sigma^{2}$ has a bounded first derivative and satisfies $\inf _{t} \sigma^{2}(t)>0$, then for $t \in(0,1)$ and $\rho>0$, uniformly for $0 \leq x \leq \rho b_{n}$,

$$
\frac{1}{1-F_{\tau}(x)} P\left( \pm \frac{n^{1 / 3}\left(\hat{\lambda}_{n}(t)-\lambda(t)\right)}{\left(4 \sigma^{2}(t)\left|\hat{\lambda}_{n}^{(1)}(t)\right|\right)^{1 / 3}} \geq x\right) \rightarrow 1 \text {. }
$$

3. Proofs of the main results. In this section, we only prove Proposition 2.1, and Theorems 2.1 and 2.2. The proofs of the results on the Grenander-type estimator are similar and will be given in Appendix A of the Supplementary Material [Gao, Xiong and Zhao (2017)]. The following two results, Theorems 3.1 and 3.2, play an important role in our proofs. Their proofs will be given in Section 5.

THEOREM 3.1. Assume that Assumptions (A1) and (A2) hold. Let $\chi_{n}$ be a sequence of nonnegative numbers such that $b_{n}^{4.5} \chi_{n} \rightarrow 0$ as $n \rightarrow \infty$.Then for each $\rho>0$, uniformly for $0 \leq x \leq \rho b_{n}$,

$$
\frac{1}{1-F_{\tau}(x)} P\left( \pm \frac{n^{1 / 3}\left(\hat{F}_{n}\left(t_{0}+\chi_{n}\right)-F_{0}\left(t_{0}+\chi_{n}\right)\right)}{\left(\sigma_{F}^{2}\left(t_{0}\right)\right)^{1 / 3}} \geq x\right) \rightarrow 1
$$

In particular, the sequence $\left\{\frac{n^{1 / 3}}{b_{n}} \frac{\hat{F}_{n}\left(t_{0}+\chi_{n}\right)-F_{0}\left(t_{0}+\chi_{n}\right)}{\left(\sigma_{F}^{2}\left(t_{0}\right)\right)^{1 / 3}}, n \geq 1\right\}$ satisfies the MDP in $\mathbb{R}$ with speed $b_{n}^{3}$ and rate function $I(x)=\frac{2}{3}|x|^{3}$.

The next result is an extension of Groeneboom and Wellner's limit distribution result. 
THEOREM 3.2. Assume that Assumption (A1) holds. Let $\chi_{n}$ be a sequence of nonnegative numbers such that $\chi_{n} \rightarrow 0$ as $n \rightarrow \infty$.Then

$$
\frac{n^{1 / 3}\left(\hat{F}_{n}\left(t_{0}+\chi_{n}\right)-F_{0}\left(t_{0}+\chi_{n}\right)\right)}{\left(\sigma_{F}^{2}\left(t_{0}\right)\right)^{1 / 3}} \stackrel{d}{\rightarrow} \tau
$$

ProOf of Proposition 2.1. Equation (2.8) is obvious. When $n$ is large enough,

$$
\begin{aligned}
\left|\hat{f}_{n}\left(t_{0}\right)-f_{0}\left(t_{0}\right)\right| \leq & \frac{1}{\log n} \frac{n^{1 / 3}}{b_{n}}\left\{\left|\hat{F}_{n}\left(t_{0}+b_{n} \log n / n^{1 / 3}\right)-F_{0}\left(t_{0}+b_{n} \log n / n^{1 / 3}\right)\right|\right. \\
& \left.+\left|\hat{F}_{n}\left(t_{0}\right)-F_{0}\left(t_{0}\right)\right|\right\} \\
& +\left|\frac{F\left(t_{0}+b_{n} \log n / n^{1 / 3}\right)-F\left(t_{0}\right)}{b_{n} \log n / n^{1 / 3}}-f_{0}\left(t_{0}\right)\right| .
\end{aligned}
$$

Since

$$
\left|\frac{F\left(t_{0}+b_{n} \log n / n^{1 / 3}\right)-F\left(t_{0}\right)}{b_{n} \log n / n^{1 / 3}}-f_{0}\left(t_{0}\right)\right| \rightarrow 0,
$$

then by Theorems 3.1, (2.9) holds. The proof of (2.10) is similar to that of (2.9).

Proof of Theorem 2.1. By Proposition 2.1(i) and the Delta method in large deviations [Gao and Zhao (2011)], for any $\epsilon>0$,

$$
\lim _{n \rightarrow \infty} \frac{1}{b_{n}^{3}} \log P\left(\frac{n^{1 / 3}}{b_{n} \log n}\left|\frac{\left(\sigma_{F}^{2}\left(t_{0}\right)\right)^{1 / 3}}{\left(\hat{\sigma}_{n}^{2}\left(t_{0}\right)\right)^{1 / 3}}-1\right|>\epsilon\right)=-\infty .
$$

For each $\rho>0$ and $0 \leq x \leq \rho b_{n}$, we have

$$
\begin{aligned}
& \frac{1}{1-F_{\tau}(x)} P\left( \pm \frac{n^{1 / 3}\left(\hat{F}_{n}\left(t_{0}\right)-F_{0}\left(t_{0}\right)\right)}{\left(\hat{\sigma}_{n}^{2}\left(t_{0}\right)\right)^{1 / 3}} \geq x\right) \\
& \leq \frac{1}{P(\tau \geq x)} P\left(\frac{n^{1 / 3}}{b_{n} \log n}\left|\frac{\left(\sigma_{F}^{2}\left(t_{0}\right)\right)^{1 / 3}}{\left(\hat{\sigma}_{n}^{2}\left(t_{0}\right)\right)^{1 / 3}}-1\right|>1\right) \\
& \quad+\frac{P\left( \pm \frac{n^{1 / 3}\left(\hat{F}_{n}\left(t_{0}\right)-F_{0}\left(t_{0}\right)\right)}{\left(\sigma_{F}^{2}\left(t_{0}\right)\right)^{1 / 3}} \geq x\left(1-\frac{b_{n} \log n}{n^{1 / 3}}\right)\right)}{P\left(\tau \geq x\left(1-\frac{b_{n} \log n}{n^{1 / 3}}\right)\right)} \frac{P\left(\tau \geq x\left(1-\frac{b_{n} \log n}{n^{1 / 3}}\right)\right)}{1-F_{\tau}(x)}
\end{aligned}
$$


and

$$
\begin{aligned}
& \frac{1}{1-F_{\tau}(x)} P\left( \pm \frac{n^{1 / 3}\left(\hat{F}_{n}\left(t_{0}\right)-F_{0}\left(t_{0}\right)\right)}{\left(\hat{\sigma}_{n}^{2}\left(t_{0}\right)\right)^{1 / 3}} \geq x\right) \\
& \geq-\frac{1}{P(\tau \geq x)} P\left(\frac{n^{1 / 3}}{b_{n} \log n}\left|\frac{\left(\sigma_{F}^{2}\left(t_{0}\right)\right)^{1 / 3}}{\left(\hat{\sigma}_{n}^{2}\left(t_{0}\right)\right)^{1 / 3}}-1\right|>1\right) \\
& \quad+\frac{P\left( \pm \frac{n^{1 / 3}\left(\hat{F}_{n}\left(t_{0}\right)-F_{0}\left(t_{0}\right)\right)}{\left(\sigma_{F}^{2}\left(t_{0}\right)\right)^{1 / 3}} \geq x\left(1+\frac{b_{n} \log n}{n^{1 / 3}}\right)\right)}{P\left(\tau \geq x\left(1+\frac{b_{n} \log n}{n^{1 / 3}}\right)\right)} \frac{P\left(\tau \geq x\left(1+\frac{b_{n} \log n}{n^{1 / 3}}\right)\right)}{1-F_{\tau}(x)}
\end{aligned}
$$

Note that by (2.2) and (3.2),

$$
\sup _{x \in\left[0, \rho b_{n}\right]} \frac{1}{P(\tau \geq x)} P\left(\frac{n^{1 / 3}}{b_{n} \log n}\left|\frac{\left(\sigma_{F}^{2}\left(t_{0}\right)\right)^{1 / 3}}{\left(\hat{\sigma}_{n}^{2}\left(t_{0}\right)\right)^{1 / 3}}-1\right|>1\right) \rightarrow 0
$$

and

$$
\sup _{0 \leq x \leq \rho b_{n}}\left|\frac{1}{1-F_{\tau}(x)} P\left(\tau \geq x\left(1+\frac{b_{n} \log n}{n^{1 / 3}}\right)\right)-1\right| \rightarrow 0 .
$$

Then (2.12) follows from (3.1) with $\chi_{n}=0$.

Proof of Theorem 2.2. By Proposition 2.1(ii) and the continuous mapping theorem [van der Vaart and Wellner (1996), page 20], for any $\epsilon>0$,

$$
\lim _{n \rightarrow \infty} P\left(\left|\frac{\left(\sigma_{F}^{2}\left(t_{0}\right)\right)^{1 / 3}}{\left(\tilde{\sigma}_{n}^{2}\left(t_{0}\right)\right)^{1 / 3}}-1\right|>\epsilon\right)=0 .
$$

Again, the conclusion follows from Theorem 3.1 with $\chi_{n}=0$.

4. Applications. In this section, we focus on applications of the selfnormalized Cramer-type MDP to statistical inference for the current status model. We also will present some simulation results to compare the finite-sample performance of the new test constructed by using the self-normalized Cramer-type MDP and the LR test [Banerjee and Wellner (2001, 2005a, 2005b), Groeneboom and Jongbloed (2015)].

4.1. Hypothesis testing. In this subsection, we apply the results in Section 2 to hypothesis testing problems for unknown distribution function $F$ in the current status model. Let $F_{0}$ and $F_{1}$ be two distributions on $[0, \infty)$ and let $t_{0} \in(0, \infty)$. Assume that $F_{0}\left(t_{0}\right) \neq F_{1}\left(t_{0}\right)$. Consider testing

$$
H_{0}: F\left(t_{0}\right)=F_{0}\left(t_{0}\right) \quad \text { versus } \quad H_{1}: F\left(t_{0}\right)=F_{1}\left(t_{0}\right) .
$$


We can use Theorem 2.1 to construct a rejection region and to prove the probability of type II error tending to 0 with exponential decay. Consider the test statistic

$$
T_{n}:=\frac{n^{1 / 3}\left(\hat{F}_{n}\left(t_{0}\right)-F_{0}\left(t_{0}\right)\right)}{\hat{\sigma}_{n}}
$$

where $\hat{\sigma}_{n}=\left(\hat{\sigma}_{n}^{2}\left(t_{0}\right)\right)^{1 / 3}$.

Given $0<\alpha<1$, by Theorem 2.1, under $H_{0}$,

$$
\lim _{n \rightarrow \infty} \sup _{x \in\left[0, \rho b_{n}\right]}\left|\frac{P\left(\left|T_{n}\right| \geq x\right)}{2\left(1-F_{\tau}(x)\right)}-1\right|=0 .
$$

Take the rejection region as $\left\{\left|T_{n}\right| \geq c(\alpha)\right\}$, where $c(\alpha)$ is a positive constant such that $\alpha / 2=1-F_{\tau}(c(\alpha))$. Then the probability $\beta_{n}$ of type II error is

$$
\beta_{n}=P\left(\left|T_{n}\right|<c(\alpha) \mid F_{1}\left(t_{0}\right)\right) .
$$

Next, we establish the exponential convergence of $\beta_{n}$.

THEOREM 4.1. Suppose that Assumptions (A1) and (A2) hold for $F_{0}$ and $F_{1}$. Then the probability of type II error tends to 0 with exponential decay speed $\exp \left\{-r b_{n}^{3}\right\}$ for all $r>0$, that is,

$$
\lim _{n \rightarrow \infty} \frac{1}{b_{n}^{3}} \log \beta_{n}=-\infty .
$$

PROOF. The probability $\beta_{n}$ of type II error is

$$
\begin{aligned}
\beta_{n} & =P\left(\left|T_{n}\right|<c(\alpha) \mid F_{1}\left(t_{0}\right)\right) \\
& \leq P\left(\left|\frac{n^{1 / 3}\left(\hat{F}_{n}\left(t_{0}\right)-F_{1}\left(t_{0}\right)\right)}{b_{n} \hat{\sigma}_{n}}\right| \geq \frac{n^{1 / 3}}{b_{n} \hat{\sigma}_{n}}\left|F_{0}\left(t_{0}\right)-F_{1}\left(t_{0}\right)\right|-\frac{c(\alpha)}{b_{n}} \mid F_{1}\left(t_{0}\right)\right) .
\end{aligned}
$$

By Proposition 2.1(i) and the Delta method in large deviations [Gao and Zhao (2011)], for any $\epsilon>0$,

$$
\lim _{n \rightarrow \infty} \frac{1}{b_{n}^{3}} \log P\left(\frac{n^{1 / 3}}{b_{n} \log n}\left|\frac{\left(\sigma_{F_{1}}^{2}\left(t_{0}\right)\right)^{1 / 3}}{\hat{\sigma}_{n}}-1\right|>\epsilon \mid F_{1}\left(t_{0}\right)\right)=-\infty .
$$

Thus, by Theorem 2.1, we have $\lim _{n \rightarrow \infty} \frac{1}{b_{n}^{3}} \log \beta_{n}=-\infty$.

4.2. Pointwise confidence interval. For given confidence level $1-\alpha$, let $\tau_{\alpha / 2}$ be a critical value such that $P\left(\tau>\tau_{\alpha / 2}\right)=\alpha / 2$.

A direct application of Theorem 2.1 yields an approximate $(1-\alpha)$ confidence interval for $F_{0}\left(t_{0}\right)$ as

$$
\left(\hat{F}_{n}\left(t_{0}\right)-\frac{\hat{\sigma}_{n}}{n^{1 / 3}} \tau_{\alpha / 2}, \hat{F}_{n}\left(t_{0}\right)+\frac{\hat{\sigma}_{n}}{n^{1 / 3}} \tau_{\alpha / 2}\right) .
$$


4.3. Simulation study. We conducted a simulation study to assess the finitesample performance of the proposed test by rejecting $H_{0}: F\left(t_{0}\right)=F_{0}\left(t_{0}\right)$ if

$$
\left|\hat{F}_{n}\left(t_{0}\right)-F_{0}\left(t_{0}\right)\right| \geq \frac{\hat{\sigma}_{n}}{n^{1 / 3}} \tau_{\alpha / 2},
$$

where $\hat{F}_{n}\left(t_{0}\right)$ is the NPMLE of $F_{0}\left(t_{0}\right)$. For $\alpha=0.05$, one can find $\tau_{0.025}=0.9982$ from Table 2 in Groeneboom and Wellner (2001) or Table I in Abrevaya and Huang (2005).

For the testing problem, other available methods include the LR, MLE-based, score and Wald test statistics [see Banerjee and Wellner (2001, 2005a, 2005b)]. Based on simulations, Banerjee and Wellner (2005a) concluded that the LR-based method is better than the MLE-based methods for the situations considered, while Banerjee and Wellner (2005b) showed that the LR and score statistics are comparable and are more powerful than the Wald statistic. Note that these so-called score test statistics are based on the difference between the unrestricted and the restricted MLEs, which can be considered as extensions of the LR test statistic. Here, we focus on comparison of the new test and the LR test. To compute the LR test statistic, we also need to find the unrestricted MLE. For this, we used the new method presented in Section 2 of Groeneboom and Jongbloed (2015).

To generate current status data, suppose that failure time $X$ follows an exponential distribution or a Weibull distribution, and examination time $T$ follows a uniform distribution over $\mathrm{i}$ an interval $\left[0, T_{0}\right]$. Let $\left\{x_{i}, i=1, \ldots, n\right\}$ and $\left\{t_{i}, i=1, \ldots, n\right\}$ be independent samples from the distributions of $X$ and $T$, respectively. The generated current status data consist of $\left\{\left(t_{i}, \delta_{i}\right), i=1, \ldots, n\right\}$ with $\delta_{i}=I_{\left(x_{i} \leq t_{i}\right)}$.

Consider two cases: (i) $F_{0}(t)=1-\exp (-0.5 t)$ and $F_{1}(t)=1-\exp (\lambda t)$ with $\lambda \neq 0.5$; and (ii) $F_{0}(t)=1-\exp \left(-(0.5 t)^{0.5}\right)$ and $F_{1}(t)=1-\exp \left(-(\lambda t)^{0.5}\right)$ with $\lambda \neq 0.5$. We set $T_{0}=2$ under $H_{0}$ and 3 or 5 under $H_{1}$.

To apply the proposed test, we need to choose $b_{n}$. Since $b_{n}$ is assumed to satisfy the following condition:

$$
b_{n} \rightarrow \infty \quad \text { and } \quad b_{n}=o\left(n^{1 / 30} /(\log n)^{1 / 10}\right),
$$

we can simply take $b_{n}=n^{1 /(30+\varepsilon)}$ with $\varepsilon>0$. According to the general principle "the bigger $b_{n}$ is, the faster the convergence rate is", we only considered values of $b_{n}$ close to $n^{1 / 30}$ in our simulations. In addition, we need to choose $h_{n}$ for estimation of $g_{0}$. As pointed out in Section 2.1, we prefer to take $h_{n}=1 / r_{n}$ where $r_{n}=n^{1 /(3+\delta)} / b_{n}$ with $\delta>0$. Here, we took values of $r_{n}$ close to $n^{1 / 3} / b_{n}$ to obtain a faster convergence speed. All the simulation results reported here are based on 1000 Monte Carlo replications using the $\mathrm{R}$ software, while the corresponding $\mathrm{R}$ code is available via the website http://www.mypolyuweb.hk/ mazhao.

Tables 1 and 2 report the estimated sizes and powers of the proposed test with $b_{n}=n^{1 / 30.1}$ and $r_{n}=n^{1 /(3.1)} / b_{n}$ and LR test at nominal level 0.05 based on simulated current status data for two cases with different values of $\lambda$. In each setting, 
TABLE 1

Estimated sizes and powers of the proposed and LR tests at nominal level 0.05 for the case of exponential distribution

\begin{tabular}{|c|c|c|c|c|c|c|c|}
\hline \multirow[b]{2}{*}{$\lambda$} & \multirow[b]{2}{*}{$T_{0}$} & \multicolumn{2}{|c|}{$n=100$} & \multicolumn{2}{|c|}{$n=200$} & \multicolumn{2}{|c|}{$n=300$} \\
\hline & & Proposed & LR & Proposed & LR & Proposed & LR \\
\hline 0.5 & 2 & 0.058 & 0.049 & 0.058 & 0.063 & 0.048 & 0.055 \\
\hline 0.7 & 3 & 0.261 & 0.157 & 0.309 & 0.227 & 0.408 & 0.324 \\
\hline 0.9 & & 0.513 & 0.427 & 0.681 & 0.645 & 0.737 & 0.732 \\
\hline 0.3 & & 0.517 & 0.386 & 0.641 & 0.558 & 0.727 & 0.682 \\
\hline 0.1 & & 1.000 & 1.000 & 1.000 & 1.000 & 1.000 & 1.000 \\
\hline 0.7 & 5 & 0.262 & 0.124 & 0.278 & 0.190 & 0.320 & 0.232 \\
\hline 0.9 & & 0.446 & 0.339 & 0.520 & 0.446 & 0.636 & 0.592 \\
\hline 0.3 & & 0.543 & 0.331 & 0.568 & 0.434 & 0.637 & 0.542 \\
\hline 0.1 & & 1.000 & 0.997 & 1.000 & 1.000 & 1.000 & 1.000 \\
\hline
\end{tabular}

we take $t_{0}=1$ and considered three sample sizes $n=100,200,300$. It can be seen from the tables that the estimated sizes using both tests are close to nominal level 0.05 , and the estimated powers of the proposed test are higher than those of the LR test for the situations considered here. Very similar results were obtained for the proposed test with other values of $b_{n}$ close to $n^{1 / 30}$ and other values of $r_{n}$ close to $n^{1 / 3} / b_{n}$ as well.

In addition, we conducted simulations to compare the power behavior of the proposed test and the LR test for the contiguous alternatives with the exponential and Weibull distributions, respectively. Let $f_{0}$ be the density function of $F_{0}$.

TABLE 2

Estimated sizes and powers of the proposed and LR tests at nominal level 0.05 for the case of Weibull distribution

\begin{tabular}{|c|c|c|c|c|c|c|c|}
\hline \multirow[b]{2}{*}{$\lambda$} & \multirow[b]{2}{*}{$T_{0}$} & \multicolumn{2}{|c|}{$n=100$} & \multicolumn{2}{|c|}{$n=200$} & \multicolumn{2}{|c|}{$n=300$} \\
\hline & & Proposed & $\mathbf{L R}$ & Proposed & $\mathbf{L R}$ & Proposed & LR \\
\hline 0.5 & 2 & 0.064 & 0.038 & 0.054 & 0.046 & 0.041 & 0.062 \\
\hline 0.7 & 3 & 0.273 & 0.079 & 0.263 & 0.116 & 0.270 & 0.138 \\
\hline 0.9 & & 0.405 & 0.215 & 0.431 & 0.290 & 0.498 & 0.389 \\
\hline 0.3 & & 0.390 & 0.239 & 0.419 & 0.320 & 0.480 & 0.390 \\
\hline 0.1 & & 0.980 & 0.964 & 1.000 & 0.999 & 0.999 & 0.999 \\
\hline 0.7 & 5 & 0.302 & 0.065 & 0.262 & 0.083 & 0.284 & 0.113 \\
\hline 0.9 & & 0.376 & 0.133 & 0.366 & 0.194 & 0.412 & 0.270 \\
\hline 0.3 & & 0.454 & 0.216 & 0.432 & 0.251 & 0.446 & 0.309 \\
\hline 0.1 & & 0.965 & 0.916 & 0.994 & 0.992 & 1.000 & 0.999 \\
\hline
\end{tabular}


Following Banerjee and Wellner (2005b), we define local alternatives $\left\{F_{n}\right\}$ as

$$
F_{n}(t)=F_{0}(t)+n^{-1 / 3} B\left(n^{1 / 3}\left(t-t_{0}\right)\right),
$$

where $B(t)=\eta f_{0}\left(t_{0}\right)(c-|t|) 1_{[-c, c]}(t)$, and $t_{0}, c, \eta$ are fixed positive numbers. Take $\eta=0.9$. The failure times can be generated by using the method given in Banerjee and Wellner (2005b). Again suppose examination time $T$ follows a uniform distribution over interval $[0,3]$. The simulation results are shown in Tables 3 and 4. The tables include the estimated powers (the rejection percentages of the null hypothesis over 1000 Monte Carlo replicates) of the proposed test and the LR test for the contiguous alternatives $H_{1}: F\left(t_{0}\right)=F_{n}\left(t_{0}\right)$ with different values of $t_{0}$ in two cases of $F_{0}$, respectively. The results indicate that the proposed test outperforms the LR test for the contiguous alternatives considered here.

We also carried out a simulation study using the Simulation Setting 3 designed by Banerjee and Wellner (2005a) with $t_{0}$ as chosen there. The results of estimated confidence intervals indicate that our method does not perform as well as the LR method for this case where the function $F_{0}$ behaves capriciously.

TABLE 3

Estimated powers of the proposed and LR tests at nominal level 0.05 for the contiguous alternatives with the exponential distribution

\begin{tabular}{|c|c|c|c|c|c|c|c|}
\hline \multirow[b]{2}{*}{$t_{0}$} & \multirow[b]{2}{*}{$c$} & \multicolumn{2}{|c|}{$n=100$} & \multicolumn{2}{|c|}{$n=200$} & \multicolumn{2}{|c|}{$n=300$} \\
\hline & & Proposed & $\mathbf{L R}$ & Proposed & LR & Proposed & LR \\
\hline \multirow[t]{5}{*}{0.5} & 1 & 0.225 & 0.060 & 0.167 & 0.071 & 0.157 & 0.059 \\
\hline & 2 & 0.284 & 0.110 & 0.230 & 0.125 & 0.232 & 0.137 \\
\hline & 3 & 0.506 & 0.361 & 0.427 & 0.325 & 0.438 & 0.342 \\
\hline & 4 & 0.734 & 0.633 & 0.705 & 0.635 & 0.689 & 0.618 \\
\hline & 5 & 0.888 & 0.837 & 0.884 & 0.870 & 0.893 & 0.870 \\
\hline \multirow[t]{5}{*}{1.0} & 1 & 0.152 & 0.052 & 0.156 & 0.053 & 0.141 & 0.075 \\
\hline & 2 & 0.225 & 0.073 & 0.191 & 0.090 & 0.177 & 0.091 \\
\hline & 3 & 0.356 & 0.192 & 0.354 & 0.189 & 0.350 & 0.188 \\
\hline & 4 & 0.559 & 0.372 & 0.532 & 0.351 & 0.546 & 0.377 \\
\hline & 5 & 0.762 & 0.606 & 0.754 & 0.580 & 0.747 & 0.600 \\
\hline \multirow[t]{5}{*}{1.5} & 1 & 0.148 & 0.048 & 0.139 & 0.054 & 0.133 & 0.055 \\
\hline & 2 & 0.225 & 0.093 & 0.195 & 0.067 & 0.173 & 0.074 \\
\hline & 3 & 0.323 & 0.121 & 0.299 & 0.132 & 0.267 & 0.124 \\
\hline & 4 & 0.491 & 0.253 & 0.486 & 0.253 & 0.463 & 0.273 \\
\hline & 5 & 0.660 & 0.432 & 0.662 & 0.466 & 0.637 & 0.435 \\
\hline \multirow[t]{5}{*}{2.5} & 1 & 0.218 & 0.062 & 0.139 & 0.059 & 0.102 & 0.056 \\
\hline & 2 & 0.288 & 0.085 & 0.197 & 0.083 & 0.158 & 0.074 \\
\hline & 3 & 0.351 & 0.142 & 0.274 & 0.141 & 0.254 & 0.128 \\
\hline & 4 & 0.441 & 0.215 & 0.337 & 0.208 & 0.299 & 0.178 \\
\hline & 5 & 0.537 & 0.353 & 0.472 & 0.327 & 0.417 & 0.328 \\
\hline
\end{tabular}


TABLE 4

Estimated powers of the proposed and LR tests at nominal level 0.05 for the contiguous alternatives with Weibull distribution

\begin{tabular}{|c|c|c|c|c|c|c|c|}
\hline \multirow[b]{2}{*}{$t_{0}$} & \multirow[b]{2}{*}{$c$} & \multicolumn{2}{|c|}{$n=100$} & \multicolumn{2}{|c|}{$n=200$} & \multicolumn{2}{|c|}{$n=300$} \\
\hline & & Proposed & $\mathbf{L R}$ & Proposed & LR & Proposed & LR \\
\hline \multirow[t]{5}{*}{0.5} & 1 & 0.208 & 0.048 & 0.182 & 0.049 & 0.171 & 0.042 \\
\hline & 2 & 0.286 & 0.068 & 0.258 & 0.075 & 0.246 & 0.075 \\
\hline & 3 & 0.448 & 0.136 & 0.382 & 0.105 & 0.369 & 0.142 \\
\hline & 4 & 0.624 & 0.324 & 0.581 & 0.275 & 0.552 & 0.245 \\
\hline & 5 & 0.752 & 0.535 & 0.759 & 0.546 & 0.756 & 0.521 \\
\hline \multirow[t]{5}{*}{1.0} & 1 & 0.212 & 0.060 & 0.212 & 0.055 & 0.184 & 0.059 \\
\hline & 2 & 0.262 & 0.058 & 0.231 & 0.057 & 0.188 & 0.054 \\
\hline & 3 & 0.343 & 0.085 & 0.291 & 0.076 & 0.283 & 0.074 \\
\hline & 4 & 0.432 & 0.112 & 0.392 & 0.125 & 0.387 & 0.138 \\
\hline & 5 & 0.536 & 0.180 & 0.541 & 0.215 & 0.523 & 0.227 \\
\hline \multirow[t]{5}{*}{1.5} & 1 & 0.198 & 0.054 & 0.192 & 0.046 & 0.187 & 0.059 \\
\hline & 2 & 0.256 & 0.061 & 0.233 & 0.040 & 0.218 & 0.050 \\
\hline & 3 & 0.294 & 0.058 & 0.258 & 0.062 & 0.247 & 0.064 \\
\hline & 4 & 0.351 & 0.091 & 0.348 & 0.094 & 0.331 & 0.113 \\
\hline & 5 & 0.445 & 0.155 & 0.440 & 0.150 & 0.422 & 0.153 \\
\hline \multirow[t]{5}{*}{2.5} & 1 & 0.223 & 0.070 & 0.142 & 0.066 & 0.100 & 0.060 \\
\hline & 2 & 0.269 & 0.082 & 0.168 & 0.079 & 0.127 & 0.063 \\
\hline & 3 & 0.272 & 0.109 & 0.210 & 0.097 & 0.183 & 0.101 \\
\hline & 4 & 0.301 & 0.124 & 0.214 & 0.122 & 0.187 & 0.109 \\
\hline & 5 & 0.310 & 0.157 & 0.245 & 0.169 & 0.208 & 0.160 \\
\hline
\end{tabular}

4.4. Conclusions. We have proposed a new test for current status data using the self-normalized Cramér-type MDP. When the size is fixed, the new test has the probability of a type II error tending to 0 with exponential decay, and thus it has good power. Our simulation study has demonstrated that the null distribution of the proposed test statistic has a reasonable approximation and that the new test is more powerful than the LR test for most of the settings considered here. However, when the true distribution function behaves capriciously, the proposed approach does not work as well as the LR method. Compared with the LR test, the new test enjoys the following advantages:

(i) The probability of type II error tends to 0 with exponential decay, which implies that the power behavior of the new test is very good.

(ii) The rejection region of the proposed test has a simple analytical expression, and thus is easy to implement. In contrast, the rejection region of the LR test must be obtained numerically.

(iii) Computationally, conducting the proposed test procedure is quite simple, but performing the LR test procedure is time-consuming. 
5. Proofs of Theorems 3.1 and 3.2. In this section, we propose a new approach to study the moderate deviation problem. Let us first give an outline of the proof. In Section 5.1, we convert the moderate deviation estimates of $\hat{F}_{n}\left(t_{0}+\chi_{n}\right)$ to those for the locations $\tau_{n}$ of minimums of a sequence of processes $Z_{n}(t), n \geq 1$. Then, in Section 5.2, we show that the processes $\left\{Z_{n}(t), n \geq 1\right\}$ can be strongly approximated by the processes $\left\{\hat{Z}_{n}(t), n \geq 1\right\}$, where $\hat{Z}_{n}(t)$ can be represented by a two-sided Wiener process plus a parabolic drift. After that, in Section 5.3, we show that $\tau_{n}$ is exponentially equivalent to the locations $\hat{\tau}_{n}$ of minimums of the processes $\hat{Z}_{n}(t)$ by the Talagrand deviation inequality and small ball estimates. The proofs of Theorems 3.1 and 3.2 are given respectively in Sections 5.4 and 5.5 using the asymptotic properties of $\hat{\tau}_{n}$.

5.1. An equivalent representation of the moderate deviations. In this subsection, we convert the moderate deviation estimates of $\hat{F}_{n}\left(t_{0}\right)$ to those of the minima of a sequence of stochastic processes.

Let $\mathbb{P}_{n}$ be the empirical measure of $\left(X_{1}, T_{1}\right), \ldots,\left(X_{n}, T_{n}\right)$, that is,

$$
\mathbb{P}_{n}(A)=\mathbb{P}_{n} I_{A}=\frac{1}{n} \sum_{i=1}^{n} I_{A}\left(X_{i}, T_{i}\right), \quad A \in \mathcal{B}\left(\mathbb{R}_{+}^{2}\right) .
$$

Set $A=\{(x, t): x \leq t\}$ and define

$$
\begin{aligned}
V_{n}(t) & =\frac{1}{n} \sum_{i=1}^{n} \delta_{i} I_{[0, t]}\left(T_{i}\right)=\mathbb{P}_{n} I_{A \cap(\mathbb{R} \times[0, t]),} \\
G_{n}(t) & =\frac{1}{n} \sum_{i=1}^{n} I_{[0, t]}\left(T_{i}\right)=\mathbb{P}_{n} I_{\mathbb{R} \times[0, t]} .
\end{aligned}
$$

The function $s \mapsto V_{n} \circ G_{n}^{-1}(s)$ equals the cumulative sum diagram [van der Vaart and Wellner (1996)], and hence, for every observation point $t_{i}$,

$$
\hat{F}_{n}\left(t_{i}\right) \leq x \text { if and only if } \underset{s}{\operatorname{argmin}}\left\{V_{n}(s)-x G_{n}(s)\right\} \geq t_{i} .
$$

Thus, the limit distribution of $\hat{F}_{n}(t)$ can be derived by studying the locations of minima of the sequence of processes $s \mapsto V_{n}(s)-x G_{n}(s)$. For each $t$, the value $\hat{F}_{n}(t)$ equals $\hat{F}_{n}\left(T_{i}\right)$ for some $i$, and hence, $\hat{F}_{n}(t) \leq x$ if and only if the argmin appearing in the display is larger than the observation time $T_{i}$ that is just left of $t$. Define $\kappa_{n}(t):=\sup \left\{1 \leq k \leq n, T_{k} \leq t\right\}$.Then

$$
\hat{F}_{n}(t) \leq x \quad \text { if and only if } \underset{s}{\operatorname{argmin}}\left\{V_{n}(s)-x G_{n}(s)\right\} \geq T_{\left(\kappa_{n}(t)\right)} .
$$

Let $\left\{\ell_{n}, n \geq 1\right\}$ be a sequence of positive numbers satisfying that

$$
1 \leq \ell_{n} \quad \text { and } \quad \lim _{n \rightarrow \infty} \frac{\ell_{n}^{10} \log n}{n^{1 / 3}}=0 .
$$


Let $\chi_{n}$ be nonnegative such that $\ell_{n}^{4.5} \chi_{n} \rightarrow 0$. Note that $\ell_{n}$ will be taken as $b_{n}$ and 1 in the proofs of Theorems 3.1 and 3.2, respectively.

Then, for any $x \in \mathbb{R}$,

$$
\begin{aligned}
& P\left(\frac{n^{1 / 3}}{\ell_{n}}\left(\hat{F}_{n}\left(t_{0}+\chi_{n}\right)-F_{0}\left(t_{0}+\chi_{n}\right)\right)>x\right) \\
& \quad=P\left(\underset{t \in\left[\left(t_{0}+\chi_{n}\right) n^{1 / 3}, \infty\right)}{\operatorname{argmin}} Y_{n}^{x}\left(t_{0}+\chi_{n}+n^{-1 / 3} t\right)<n^{1 / 3}\left(T_{\left(\bar{\kappa}_{n}\right)}-\left(t_{0}+\chi_{n}\right)\right)\right),
\end{aligned}
$$

where $\bar{\kappa}_{n}=\kappa_{n}\left(t_{0}+\chi_{n}\right)$ and $Y_{n}^{x}(s)=V_{n}(s)-\left(F_{0}\left(t_{0}+\chi_{n}\right)+n^{-1 / 3} \ell_{n} x\right) G_{n}(s)$.

Note that the decomposition of $Y_{n}^{x}\left(t_{0}+\chi_{n}+n^{-1 / 3} t\right)$ on page 299 of van der Vaart and Wellner (1996) is not suitable for the application of strong approximation. In order to apply the strong approximation, we write $Y_{n}^{x}\left(t_{0}+\chi_{n}+n^{-1 / 3} t\right)$ as

$$
Z_{n}^{x}(t)=U_{n}(t)+r_{n}(t)+x \ell_{n} \gamma_{n}(t)+R_{n}(t)+x \ell_{n} \Gamma_{n}(t)
$$

with

$$
\left\{\begin{aligned}
U_{n}(t) & :=\frac{1}{n^{1 / 3}} \sum_{i=1}^{n}\left(I_{\left\{X_{i} \leq t_{0}+\chi_{n}\right\}}-F_{0}\left(t_{0}+\chi_{n}\right)\right)\left(I_{\left\{T_{i} \leq t_{0}+\chi_{n}+n^{-1 / 3} t\right\}}-I_{\left\{T_{i} \leq t_{0}+\chi_{n}\right\}}\right), \\
r_{n}(t) & :=n^{2 / 3} E\left(\left(I_{\left\{X_{1} \leq T_{1}\right\}}-I_{\left\{X_{1} \leq t_{0}+\chi_{n}\right\}}\right)\left(I_{\left\{T_{1} \leq t_{0}+\chi_{n}+n^{-1 / 3} t\right\}}-I_{\left\{T_{1} \leq t_{0}+\chi_{n}\right\}}\right)\right) \\
\gamma_{n}(t) & :=n^{1 / 3}\left(G\left(t_{0}+\chi_{n}+n^{-1 / 3} t\right)-G\left(t_{0}+\chi_{n}\right)\right) \\
R_{n}(t) & :=\frac{1}{n^{1 / 3}} \sum_{i=1}^{n}\left(I_{\left\{X_{i} \leq T_{i}\right\}}-I_{\left\{X_{i} \leq t_{0}+\chi_{n}\right\}}\right)\left(I_{\left\{T_{i} \leq t_{0}+\chi_{n}+n^{-1 / 3} t\right\}}-I_{\left\{T_{i} \leq t_{0}+\chi_{n}\right\}}\right)-r_{n}(t) \\
\Gamma_{n}(t) & :=\frac{1}{n^{2 / 3}} \sum_{i=1}^{n}\left(I_{\left\{T_{i} \leq t_{0}+\chi_{n}+n^{-1 / 3} t\right\}}-I_{\left\{T_{i} \leq t_{0}+\chi_{n}\right\}}\right)-\gamma_{n}(t)
\end{aligned}\right.
$$

This decomposition plays a major role in the proof of our main result. Therefore,

$$
\begin{aligned}
& P\left(\frac{n^{1 / 3}}{\ell_{n}}\left(\hat{F}_{n}\left(t_{0}+\chi_{n}\right)-F_{0}\left(t_{0}+\chi_{n}\right)\right)>x\right) \\
& \quad=P\left(\underset{t \in\left[-\left(t_{0}+\chi_{n}\right) n^{1 / 3}, \infty\right)}{\operatorname{argmin}} Z_{n}^{-x}(t)<n^{1 / 3}\left(T_{\left(\bar{\kappa}_{n}\right)}-\left(t_{0}+\chi_{n}\right)\right)\right) .
\end{aligned}
$$

Similarly, for any $x \in \mathbb{R}$,

$$
\begin{aligned}
& P\left(\frac{n^{1 / 3}}{\ell_{n}}\left(\hat{F}_{n}\left(t_{0}+\chi_{n}\right)-F_{0}\left(t_{0}+\chi_{n}\right)\right) \leq-x\right) \\
& \quad=P\left(\underset{t \in\left[-\left(t_{0}+\chi_{n}\right) n^{1 / 3}, \infty\right)}{\operatorname{argmin}} Z_{n}^{x}(t) \geq n^{1 / 3}\left(T_{\left(\bar{\kappa}_{n}\right)}-\left(t_{0}+\chi_{n}\right)\right)\right) .
\end{aligned}
$$


LEMMA 5.1. For any $\delta>0$,

$$
\limsup _{n \rightarrow \infty} \frac{1}{\ell_{n}^{3}} \log P\left(n^{1 / 3}\left(t_{0}+\chi_{n}-T_{\left(\bar{\kappa}_{n}\right)}\right) \geq \ell_{n}^{-2} \delta\right)=-\infty .
$$

PROOF. For any $\delta>0$,

$$
\begin{aligned}
& P\left(n^{1 / 3}\left(t_{0}+\chi_{n}-T_{\left(\bar{\kappa}_{n}\right)}\right) \geq \ell_{n}^{-2} \delta\right) \\
& \quad=P\left(T_{i} \notin\left(t_{0}+\chi_{n}-n^{-1 / 3} \ell_{n}^{-2} \delta, t_{0}+\chi_{n}\right) \text { for all } i=1, \ldots, n\right) \\
& \quad=\left(1-\int_{t_{0}+\chi_{n}-n^{-1 / 3} \ell_{n}^{-2} \delta}^{t_{0}+\chi_{n}} g(t) d t\right)^{n} .
\end{aligned}
$$

Thus,

$$
\limsup _{n \rightarrow \infty} \frac{1}{\ell_{n}^{3}} \log P\left(n^{1 / 3}\left(t_{0}+\chi_{n}-T_{\left(\bar{\kappa}_{n}\right)}\right) \geq \ell_{n}^{-2} \delta\right)=-\infty .
$$

Proposition 5.1. For each $\rho>0$, we have

$$
\begin{aligned}
& \limsup _{n \rightarrow \infty} \sup _{x \in[0, \rho]} \frac{P\left(n^{1 / 3}\left(\hat{F}_{n}\left(t_{0}+\chi_{n}\right)-F_{0}\left(t_{0}+\chi_{n}\right)\right)>x \ell_{n}\right)}{1-F_{\tau}\left(x\left(\sigma_{F}^{2}\left(t_{0}\right)\right)^{-1 / 3} \ell_{n}\right)} \\
& \quad \leq \limsup _{n \rightarrow \infty} \sup _{x \in[0, \rho]} \frac{P\left(\operatorname{argmin}_{t \in\left[-\left(t_{0}+\chi_{n}\right) n^{1 / 3}, \infty\right)} Z_{n}^{-x}(t) \leq 0\right)}{1-F_{\tau}\left(x\left(\sigma_{F}^{2}\left(t_{0}\right)\right)^{-1 / 3} \ell_{n}\right)},
\end{aligned}
$$

$$
\liminf _{n \rightarrow \infty} \inf _{x \in[0, \rho]} \frac{P\left(n^{1 / 3}\left(\hat{F}_{n}\left(t_{0}+\chi_{n}\right)-F_{0}\left(t_{0}+\chi_{n}\right)\right)>x \ell_{n}\right)}{1-F_{\tau}\left(x\left(\sigma_{F}^{2}\left(t_{0}\right)\right)^{-1 / 3} \ell_{n}\right)}
$$

$$
\geq \lim _{\delta \downarrow 0} \liminf _{n \rightarrow \infty} \inf _{x \in[0, \rho]} \frac{P\left(\operatorname{argmin}_{t \in\left[-\left(t_{0}+\chi_{n}\right) n^{1 / 3}, \infty\right)} Z_{n}^{-x}(t) \leq-\ell_{n}^{-2} \delta\right)}{1-F_{\tau}\left(x\left(\sigma_{F}^{2}\left(t_{0}\right)\right)^{-1 / 3} \ell_{n}\right)},
$$

$$
\begin{array}{r}
\liminf _{n \rightarrow \infty} \inf _{x \in[0, \rho]} \frac{P\left(n^{1 / 3}\left(\hat{F}_{n}\left(t_{0}+\chi_{n}\right)-F_{0}\left(t_{0}+\chi_{n}\right)\right) \leq-x \ell_{n}\right)}{1-F_{\tau}\left(x\left(\sigma_{F}^{2}\left(t_{0}\right)\right)^{-1 / 3} \ell_{n}\right)} \\
\quad \geq \liminf _{n \rightarrow \infty} \inf _{x \in[0, \rho]} \frac{P\left(\operatorname{argmin}_{t \in\left[-\left(t_{0}+\chi_{n}\right) n^{1 / 3}, \infty\right)} Z_{n}^{x}(t) \geq 0\right)}{1-F_{\tau}\left(x\left(\sigma_{F}^{2}\left(t_{0}\right)\right)^{-1 / 3} \ell_{n}\right)}
\end{array}
$$

and

$$
\begin{aligned}
& \limsup _{n \rightarrow \infty} \sup _{x \in[0, \rho]} \frac{P\left(n^{1 / 3}\left(\hat{F}_{n}\left(t_{0}+\chi_{n}\right)-F_{0}\left(t_{0}+\chi_{n}\right)\right) \leq-x \ell_{n}\right)}{1-F_{\tau}\left(x\left(\sigma_{F}^{2}\left(t_{0}\right)\right)^{-1 / 3} \ell_{n}\right)} \\
& \quad \leq \lim _{\delta \downarrow 0} \limsup _{n \rightarrow \infty} \sup _{x \in[0, \rho]} \frac{P\left(\operatorname{argmin}_{t \in\left[-\left(t_{0}+\chi_{n}\right) n^{1 / 3}, \infty\right)} Z_{n}^{x}(t) \geq-\ell_{n}^{-2} \delta\right)}{1-F_{\tau}\left(x\left(\sigma_{F}^{2}\left(t_{0}\right)\right)^{-1 / 3} \ell_{n}\right)} .
\end{aligned}
$$


PROOF. For each $\rho>0$ and $0 \leq x \leq \rho$, we have

$$
\begin{aligned}
& \frac{P\left(\operatorname{argmin}_{t \in\left[-\left(t_{0}+\chi_{n}\right) n^{1 / 3}, \infty\right)} Z_{n}^{-x}(t) \leq-\ell_{n}^{-2} \delta\right)-P\left(n^{1 / 3}\left(t_{0}+\chi_{n}-T_{\left(\bar{\kappa}_{n}\right)}\right) \geq \ell_{n}^{-2} \delta\right)}{1-F_{\tau}\left(x\left(\sigma_{F}^{2}\left(t_{0}\right)\right)^{-1 / 3} \ell_{n}\right)} \\
& \quad \leq \frac{P\left(n^{1 / 3}\left(\hat{F}_{n}\left(t_{0}+\chi_{n}\right)-F_{0}\left(t_{0}+\chi_{n}\right)\right)>x \ell_{n}\right)}{1-F_{\tau}\left(x\left(\sigma_{F}^{2}\left(t_{0}\right)\right)^{-1 / 3} \ell_{n}\right)} \\
& \leq \frac{P\left(\operatorname{argmin}_{t \in\left[-\left(t_{0}+\chi_{n}\right) n^{1 / 3}, \infty\right)} Z_{n}^{-x}(t) \leq 0\right)}{1-F_{\tau}\left(x\left(\sigma_{F}^{2}\left(t_{0}\right)\right)^{-1 / 3} \ell_{n}\right)}
\end{aligned}
$$

and

$$
\begin{aligned}
& \frac{P\left(\operatorname{argmin}_{t \in\left[-\left(t_{0}+\chi_{n}\right) n^{1 / 3}, \infty\right)} Z_{n}^{x}(t) \geq 0\right)}{1-F_{\tau}\left(x\left(\sigma_{F}^{2}\left(t_{0}\right)\right)^{-1 / 3} \ell_{n}\right)} \\
& \quad \leq \frac{P\left(n^{1 / 3}\left(\hat{F}_{n}\left(t_{0}+\chi_{n}\right)-F_{0}\left(t_{0}+\chi_{n}\right)\right) \leq-x \ell_{n}\right)}{1-F_{\tau}\left(x\left(\sigma_{F}^{2}\left(t_{0}\right)\right)^{-1 / 3} \ell_{n}\right)} \\
& \quad \leq \frac{P\left(\operatorname{argmin}_{t \in\left[-\left(t_{0}+\chi_{n}\right) n^{1 / 3}, \infty\right)} Z_{n}^{x}(t) \geq-\ell_{n}^{-2} \delta\right)+P\left(n^{1 / 3}\left(t_{0}+\chi_{n}-T_{\left(\bar{\kappa}_{n}\right)}\right) \geq \ell_{n}^{-2} \delta\right)}{1-F_{\tau}\left(x\left(\sigma_{F}^{2}\left(t_{0}\right)\right)^{-1 / 3} \ell_{n}\right)} .
\end{aligned}
$$

Therefore, by Lemma 5.1, we obtain the conclusions of the proposition.

5.2. A strong approximation theorem associated with the NPMLE. In this subsection, we present an exponential approximation for a version of $U_{n}(t)$ by a two-sided Wiener process. A similar approximation is also derived for the process $Z_{n}^{x}(t)$. This estimate will play a pivotal role in our proof of the moderate deviation result.

LEMMA 5.2. Let $Y_{i}=I_{\left\{X_{i} \leq t_{0}+\chi_{n}\right\}}-F_{0}\left(t_{0}+\chi_{n}\right)$. There exist a version $\left(\left\{Y_{i}^{*}, i \geq 1\right\},\left\{T_{i}^{*}, i \geq 1\right\}\right)$ of $\left(\left\{Y_{i}, i \geq 1\right\},\left\{T_{i}, i \geq 1\right\}\right)$, a two-sided Wiener process $W_{n}(t)$ with $W_{n}(0)=0, n \geq 1$ and two positive absolute constants $C, K$ such that for any $\delta>0$,

$$
\begin{aligned}
& P\left(\sup _{t \in\left[-\left(t_{0}+\chi_{n}\right) n^{1 / 3}, \infty\right)}\left|U_{n}^{*}(t)-n^{1 / 6} \sigma_{U} W_{n}\left(G_{n}^{*}(t)\right)\right| \geq \delta\right) \\
& \quad \leq \frac{n^{K C}}{K} \exp \left\{-K \delta n^{1 / 3}\right\},
\end{aligned}
$$

where

$$
\begin{aligned}
& U_{n}^{*}(t):=\frac{1}{n^{1 / 3}} \sum_{i=1}^{n} Y_{i}^{*} I_{\left\{t_{0}+\chi_{n}<T_{i}^{*} \leq t_{0}+\chi_{n}+n^{-1 / 3} t\right\}}, \\
& G_{n}^{*}(t)=G\left(t_{0}+\chi_{n}+n^{-1 / 3} t\right)-G\left(t_{0}+\chi_{n}\right)
\end{aligned}
$$

and

$$
\sigma_{U}^{2}=E\left(\left(I_{\left\{X_{i} \leq t_{0}+\chi_{n}\right\}}-F_{0}\left(t_{0}+\chi_{n}\right)\right)^{2}\right)=F_{0}\left(t_{0}+\chi_{n}\right)\left(1-F_{0}\left(t_{0}+\chi_{n}\right)\right) .
$$


PROOF. Since for any two-sided Wiener process $W_{n}$ with $W_{n}(0)=0$,

$$
\left\{W_{n}\left(G\left(t_{0}+\chi_{n}+n^{-1 / 3} t\right)\right)-W_{n}\left(G\left(t_{0}+\chi_{n}\right), t \in \mathbb{R}\right\} \stackrel{d}{=}\left\{W_{n}\left(G_{n}^{*}(t)\right), t \in \mathbb{R}\right\},\right.
$$

applying Theorem 5 in Maumy (2004) or Theorem B.3 in Appendix B of the Supplementary Material [Gao, Xiong and Zhao (2017)] to $Y_{i}$ and $Z_{i}=G\left(T_{i}\right)$, there exist a version $\left(\left\{Y_{i}^{*}, i \geq 1\right\},\left\{T_{i}^{*}, i \geq 1\right\}\right)$ of $\left(\left\{Y_{i}, i \geq 1\right\},\left\{T_{i}, i \geq 1\right\}\right)$, a Wiener process $W_{n}(t), n \geq 1$ and two positive absolute constants $C, K$ such that for all $\delta>0$, (5.6) holds.

We can write

$$
\left\{\begin{array}{l}
R_{n}(t)=\sum_{i=1}^{n} \frac{1}{n}\left(g_{n}\left(X_{i}, T_{i}, t\right)-r_{n}(t)\right)=: \sum_{i=1}^{n} Z_{n i}(t) \\
\Gamma_{n}(t)=\sum_{i=1}^{n} \frac{1}{n}\left(h_{n}\left(T_{i}, t\right)-\gamma_{n}(t)\right)=: \sum_{i=1}^{n} \xi_{n i}(t)
\end{array}\right.
$$

where

$$
\left\{\begin{aligned}
g_{n}(y, u, t) & =n^{2 / 3}\left(I_{\{y \leq u\}}-I_{\left\{y \leq t_{0}+\chi_{n}\right\}}\right)\left(I_{\left\{u \leq t_{0}+\chi_{n}+n^{-1 / 3} t\right\}}-I_{\left\{u \leq t_{0}+\chi_{n}\right\}}\right), \\
h_{n}(u, t) & =n^{1 / 3}\left(I_{\left\{u \leq t_{0}+\chi_{n}+n^{-1 / 3} t\right\}}-I_{\left\{u \leq t_{0}+\chi_{n}\right\}}\right) .
\end{aligned}\right.
$$

When $t \in\left[-\epsilon_{0} n^{1 / 3} / 2, \epsilon_{0} n^{1 / 3} / 2\right]$,

$$
\left\{\begin{array}{l}
r_{n}(t)=\int_{0}^{t} \int_{0}^{u} f_{0}\left(t_{0}+\chi_{n}+n^{-1 / 3} s\right) d s g\left(t_{0}+\chi_{n}+n^{-1 / 3} u\right) d u \\
\gamma_{n}(t)=\int_{0}^{t} g\left(t_{0}+\chi_{n}+n^{-1 / 3} u\right) d u
\end{array}\right.
$$

The following lemma is straightforward.

LEMMA 5.3. (i) If $\ell_{n}=1$ for all $n \geq 1$, then, under Assumption (A1),

$$
\left\{\begin{array}{l}
\lim _{n \rightarrow \infty} \sup _{|t| \leq N}\left|r_{n}(t)-\frac{f_{0}\left(t_{0}\right) g\left(t_{0}\right) t^{2}}{2}\right|=0 \\
\lim _{n \rightarrow \infty} \sup _{|t| \leq N}\left|\gamma_{n}(t)-g\left(t_{0}\right) t\right|=0
\end{array}\right.
$$

(ii) If Assumptions (A1) and (A2) hold, then there exists a positive constant $L$ such that, for any $N \geq 1$,

$$
\left\{\begin{array}{l}
\sup _{|t| \leq N \ell_{n}}\left|r_{n}(t)-\frac{f_{0}\left(t_{0}\right) g\left(t_{0}\right) t^{2}}{2}\right| \leq L\left(n^{-1 / 3} N^{3} \ell_{n}^{3}+n^{-2 / 3} N^{4} \ell_{n}^{4}+N^{2} \ell_{n}^{2} \chi_{n}\right), \\
\sup _{|t| \leq N \ell_{n}}\left|\gamma_{n}(t)-g\left(t_{0}\right) t\right| \leq L\left(N^{2} \ell_{n}^{2} n^{-1 / 3}+N \ell_{n} \chi_{n}\right) .
\end{array}\right.
$$

In particular, (5.7) holds. 
Set

$$
\begin{cases}\sigma_{R, n, \lambda}^{2}=E\left(\sup _{t \in[-1,1]} \sum_{i=1}^{n}\left|Z_{n i}(\lambda t)\right|^{2}\right), & \mu_{R, n, \lambda}=E\left(\sup _{t \in[-1,1]}\left|R_{n}(\lambda t)\right|\right), \\ \sigma_{\Gamma, n, \lambda}^{2}=E\left(\sup _{t \in[-1,1]} \sum_{i=1}^{n}\left|\xi_{n i}(\lambda t)\right|^{2}\right), & \mu_{\Gamma, n, \lambda}=E\left(\sup _{t \in[-1,1]}\left|\Gamma_{n}(\lambda t)\right|\right) .\end{cases}
$$

Then by Proposition 2.1 in Giné and Guillou (2001) or Lemma B.1 in Appendix B of the Supplementary Material [Gao, Xiong and Zhao (2017)], there exists a positive constant $L$ such that

$$
\begin{cases}\sigma_{R, n, N \ell_{n}}^{2} \leq L N^{2} \ell_{n}^{2} n^{-1 / 3}, & \mu_{R, n, N \ell_{n}} \leq L N \ell_{n} n^{-1 / 6}(\log n)^{1 / 2} \\ \sigma_{\Gamma, n, N \ell_{n}}^{2} \leq L \ell_{n} N n^{-2 / 3}, & \mu_{\Gamma, n, N \ell_{n}} \leq L N^{1 / 2} \ell_{n}^{1 / 2} n^{-1 / 3}(\log n)^{1 / 2} .\end{cases}
$$

Now, define

$$
\begin{gathered}
\tilde{Z}_{n}^{x}(t)=U_{n}(t)+\frac{f_{0}\left(t_{0}\right) g\left(t_{0}\right) t^{2}}{2}+x \ell_{n} g\left(t_{0}\right) t, \\
\tilde{Z}_{n}^{x, *}(t)=U_{n}^{*}(t)+\frac{f_{0}\left(t_{0}\right) g\left(t_{0}\right) t^{2}}{2}+x \ell_{n} g\left(t_{0}\right) t
\end{gathered}
$$

and

$$
\hat{Z}_{n}^{x}(t)=n^{1 / 6} \sigma_{U} W_{n}\left(G_{n}^{*}(t)\right)+\frac{f_{0}\left(t_{0}\right) g\left(t_{0}\right) t^{2}}{2}+x \ell_{n} g\left(t_{0}\right) t
$$

Then the processes $\tilde{Z}_{n}^{x}$ and $\tilde{Z}_{n}^{x, *}$ have the same distribution.

Proposition 5.2. Suppose Assumption (A1) holds. If $\ell_{n}=1$ for all $n \geq 1$, or Assumption (A2) holds, then for any $\delta>0$ and $\rho>0$,

$$
\limsup _{n \rightarrow \infty} \frac{1}{\ell_{n}^{3}} \log \sup _{|x| \in[0, \rho]} P\left(\ell_{n}^{5 / 2} \sup _{|t| \leq N \ell_{n}}\left|Z_{n}^{x}(t)-\tilde{Z}_{n}^{x}(t)\right| \geq \delta\right)=-\infty
$$

and

$$
\limsup _{n \rightarrow \infty} \frac{1}{\ell_{n}^{3}} \log \sup _{|x| \in[0, \rho]} P\left(\ell_{n}^{5 / 2} \sup _{|t| \leq N \ell_{n}}\left|\tilde{Z}_{n}^{x, *}(t)-\hat{Z}_{n}^{x}(t)\right| \geq \delta\right)=-\infty
$$

PROOF. First, by (5.6),

$\limsup _{n \rightarrow \infty} \frac{1}{\ell_{n}^{3}} \log P\left(\ell_{n}^{5 / 2} \sup _{t \in\left[-\left(t_{0}+\chi_{n}\right) n^{1 / 3}, \infty\right)}\left|U_{n}^{*}(t)-n^{1 / 6} \sigma_{U} W_{n}\left(G_{n}^{*}(t)\right)\right| \geq \delta\right)=-\infty$.

Thus, (5.10) is valid.

Applying Talagrand's inequality (see Theorem 1.4 in Talagrand (1996), or Theorem B.1 in Appendix B of the Supplementary Material [Gao, Xiong and Zhao 
(2017)]) to $\left\{n^{1 / 3} R_{n}\left(N \ell_{n} t\right) ; t \in[-1,1]\right\}$ and $\left\{n^{1 / 3} \ell_{n} \Gamma_{n}\left(N \ell_{n} t\right) ; t \in[-1,1]\right\}$, there exists a constant $L \in(0, \infty)$ such that for all $n \geq 1$ and any $\delta>0$,

$$
\begin{aligned}
& P\left(\ell_{n}^{5 / 2}\left|\sup _{|t| \leq N \ell_{n}}\right| R_{n}(t)\left|-E\left(\sup _{|t| \leq N \ell_{n}}\left|R_{n}(t)\right|\right)\right| \geq \delta\right) \\
& \quad \leq L \exp \left\{-\frac{\delta n^{1 / 3} / \ell_{n}^{5 / 2}}{L} \log \left(1+\frac{L \delta / \ell_{n}^{5 / 2}}{n^{1 / 3} \sigma_{R, n, N \ell_{n}}^{2}}\right)\right\}
\end{aligned}
$$

and

$$
\begin{aligned}
& P\left(\ell_{n}^{7 / 2}\left|\sup _{|t| \leq N \ell_{n}}\right| \Gamma_{n}(t)\left|-E\left(\sup _{|t| \leq N \ell_{n}}\left|\Gamma_{n}(t)\right|\right)\right| \geq \delta\right) \\
& \quad=P\left(\ell_{n}\left|\sup _{t \in[-1,1]}\right| n^{1 / 3} \Gamma_{n}\left(N \ell_{n} t\right)\left|-E\left(\sup _{t \in[-1,1]}\left|n^{1 / 3} \Gamma_{n}\left(N \ell_{n} t\right)\right|\right)\right| \geq \delta n^{1 / 3} / \ell_{n}^{5 / 2}\right) \\
& \quad \leq L \exp \left\{-\frac{\delta n^{1 / 3} / \ell_{n}^{5 / 2}}{L} \log \left(1+\frac{L \delta / \ell_{n}^{5 / 2}}{n^{1 / 3} \ell_{n}^{2} \sigma_{\Gamma, n, N \ell_{n}}^{2}}\right)\right\} .
\end{aligned}
$$

Therefore, by (5.8), if $\ell_{n}=1$ for all $n \geq 1$, or Assumption (A2) holds, then

$$
\limsup _{n \rightarrow \infty} \frac{1}{\ell_{n}^{3}} \log P\left(\ell_{n}^{5 / 2} \sup _{|t| \leq N \ell_{n}}\left|R_{n}(t)\right| \geq \delta\right)=-\infty
$$

and

$$
\limsup _{n \rightarrow \infty} \frac{1}{\ell_{n}^{3}} \log P\left(\ell_{n}^{7 / 2} \sup _{|t| \leq N \ell_{n}}\left|\Gamma_{n}(t)\right| \geq \delta\right)=-\infty .
$$

These yield (5.9).

5.3. Exponential equivalence. For each $x \in \mathbb{R}$, let

$$
\left\{\begin{aligned}
\tau_{n}^{x} & =\underset{t \in\left[-\left(t_{0}+\chi_{n}\right) n^{1 / 3}, \infty\right)}{\operatorname{argmin}} Z_{n}^{x}(t), \\
\hat{\tau}_{n}^{x} & =\underset{t \in\left[-\left(t_{0}+\chi_{n}\right) n^{1 / 3}, \infty\right)}{\operatorname{argmin}} \hat{Z}_{n}^{x}(t), \\
\tilde{\tau}_{n}^{x} & =\underset{t \in\left[-\left(t_{0}+\chi_{n}\right) n^{1 / 3}, \infty\right)}{\operatorname{argmin}} \tilde{Z}_{n}^{x}(t), \\
\tilde{\tau}_{n}^{x, *} & =\underset{t \in\left[-\left(t_{0}+\chi_{n}\right) n^{1 / 3}, \infty\right)}{\operatorname{argmin}} \tilde{Z}_{n}^{x, *}(t) .
\end{aligned}\right.
$$

Then the processes $\tilde{\tau}_{n}^{x}$ and $\tilde{\tau}_{n}^{x, *}$ have the same distribution.

The main result in this subsection is the following exponential equivalence theorem. 
Proposition 5.3. Suppose Assumption (A1) holds. If $\ell_{n}=1$ for all $n \geq 1$, or Assumption (A2) holds with $\ell_{n} \rightarrow \infty$, then for any $\delta>0$ and $\rho>0$,

$$
\limsup _{n \rightarrow \infty} \frac{1}{\ell_{n}^{3}} \log \sup _{|x| \in[0, \rho]} P\left(\left|\tilde{\tau}_{n}^{x, *}-\hat{\tau}_{n}^{x}\right| \geq \ell_{n}^{-2} \delta\right)=-\infty
$$

and

$$
\limsup _{n \rightarrow \infty} \frac{1}{\ell_{n}^{3}} \log \sup _{|x| \in[0, \rho]} P\left(\left|\tau_{n}^{x}-\tilde{\tau}_{n}^{x}\right| \geq \ell_{n}^{-2} \delta\right)=-\infty .
$$

Proposition 5.3 will be proved through the following two lemmas. Their proofs are based on the Talagrand deviation inequality and small ball estimates, and will be given in Appendix A of the Supplementary Material [Gao, Xiong and Zhao (2017)].

LEMMA 5.4. For each $\rho>0$,

$$
\limsup _{N \rightarrow \infty} \limsup _{n \rightarrow \infty} \frac{1}{\ell_{n}^{3}} \log \sup _{|x| \in[0, \rho]} P\left(\max \left\{\left|\tilde{\tau}_{n}^{x}\right|,\left|\tau_{n}^{x}\right|\right\} \geq \ell_{n} N\right)=-\infty
$$

and

$$
\underset{N \rightarrow \infty}{\limsup } \limsup _{n \rightarrow \infty} \frac{1}{\ell_{n}^{3}} \log \sup _{|x| \in[0, \rho]} P\left(\max \left\{\left|\hat{\tau}_{n}^{x}\right|,\left|\tilde{\tau}_{n}^{x, *}\right|\right\} \geq \ell_{n} N\right)=-\infty
$$

LEMMA 5.5. For each $\rho>0, \delta>0$ and $N \geq 1$,

$$
\lim _{\epsilon \rightarrow 0} \limsup _{n \rightarrow \infty} \frac{1}{\ell_{n}^{3}} \log \sup _{|x| \in[0, \rho]} P\left(\left|\hat{\tau}_{n}^{x}\right| \leq N \ell_{n}, \hat{Z}_{n}^{x}\left(\hat{\tau}_{n}^{x}\right)-\inf _{\substack{|s| \leq N \ell_{n},\left|s-\hat{\tau}_{n}^{x}\right| \geq \delta \ell_{n}^{-2}}} \hat{Z}_{n}^{x}(s) \geq \frac{-\epsilon}{\ell_{n}^{5 / 2}}\right)
$$

$$
=-\infty
$$

and

$$
\begin{aligned}
& \lim _{\epsilon \rightarrow 0} \limsup _{n \rightarrow \infty} \frac{1}{\ell_{n}^{3}} \log \sup _{|x| \in[0, \rho]} P\left(\left|\tilde{\tau}_{n}^{x}\right| \leq N \ell_{n}, \tilde{Z}_{n}^{x}\left(\tilde{\tau}_{n}^{x}\right)-\inf _{\substack{|s| \leq N \ell_{n},\left|s-\tilde{\tau}_{n}^{x}\right| \geq \delta \ell_{n}^{-2}}} \tilde{Z}_{n}^{x}(s) \geq \frac{-\epsilon}{\ell_{n}^{5 / 2}}\right) \\
& \quad=-\infty .
\end{aligned}
$$

Finally, we complete the proof of Proposition 5.3.

Proof of Proposition 5.3. We only show (5.11). For any $\delta>0, N \geq 1$, and $\epsilon \in(0,1 / 2)$, set

$$
\Delta_{n}=\hat{Z}_{n}^{x}\left(\hat{\tau}_{n}^{x}\right)-\inf _{|s| \leq N \ell_{n},\left|s-\hat{\tau}_{n}^{x}\right| \geq \delta \ell_{n}^{-2}} \hat{Z}_{n}^{x}(s) .
$$


Note that on $\left\{\left|\tilde{\tau}_{n}^{x, *}\right| \leq \ell_{n} N\right\}$, for any $|s| \leq \ell_{n} N$,

$$
\begin{aligned}
\hat{Z}_{n}^{x}(s)-\hat{Z}_{n}^{x}\left(\tilde{\tau}_{n}^{x, *}\right) & =\hat{Z}_{n}^{x}(s)-\tilde{Z}_{n}^{x, *}\left(\tilde{\tau}_{n}^{x, *}\right)+\tilde{Z}_{n}^{x, *}\left(\tilde{\tau}_{n}^{x, *}\right)-\hat{Z}_{n}^{x}\left(\tilde{\tau}_{n}^{x, *}\right) \\
& \geq \hat{Z}_{n}^{x}(s)-\tilde{Z}_{n}^{x, *}(s)+\tilde{Z}_{n}^{x, *}\left(\tilde{\tau}_{n}^{x, *}\right)-\hat{Z}_{n}^{x}\left(\tilde{\tau}_{n}^{x, *}\right) \\
& \geq-2 \sup _{|s| \leq \ell_{n} N}\left|\hat{Z}_{n}^{x}(s)-\tilde{Z}_{n}^{x, *}(s)\right| .
\end{aligned}
$$

Then

$$
\begin{aligned}
& P\left(\left|\tilde{\tau}_{n}^{x, *}-\hat{\tau}_{n}^{x}\right| \geq \ell_{n}^{-2} \delta\right) \\
& \leq P\left(\max \left\{\left|\tilde{\tau}_{n}^{x, *}\right|,\left|\hat{\tau}_{n}^{x}\right|\right\} \geq N \ell_{n}\right)+P\left(\left|\hat{\tau}_{n}^{x}\right| \leq N \ell_{n}, \Delta_{n} \geq-\epsilon \mid \ell_{n}^{5 / 2}\right) \\
&+P\left(\max \left\{\left|\tilde{\tau}_{n}^{x, *}\right|,\left|\hat{\tau}_{n}^{x}\right|\right\} \leq N \ell_{n}, \ell_{n}^{5 / 2} \Delta_{n}<-\epsilon,\left|\tilde{\tau}_{n}^{x, *}-\hat{\tau}_{n}^{x}\right| \geq \ell_{n}^{-2} \delta\right) \\
& \leq P\left(\max \left\{\left|\tilde{\tau}_{n}^{x, *}\right|,\left|\hat{\tau}_{n}^{x}\right|\right\} \geq N \ell_{n}\right)+P\left(\left|\hat{\tau}_{n}\right| \leq N \ell_{n}, \Delta_{n} \geq-\epsilon / \ell_{n}^{5 / 2}\right) \\
&+P\left(\max \left\{\left|\tilde{\tau}_{n}^{x, *}\right|,\left|\hat{\tau}_{n}^{x}\right|\right\} \leq N \ell_{n}, \ell_{n}^{5 / 2}\left(\hat{Z}_{n}^{x}\left(\hat{\tau}_{n}^{x}\right)-\hat{Z}_{n}^{x}\left(\tilde{\tau}_{n}^{x, *}\right)\right)<-\epsilon\right) \\
& \leq P\left(\max \left\{\left|\tilde{\tau}_{n}^{x, *}\right|,\left|\hat{\tau}_{n}^{x}\right|\right\} \geq N \ell_{n}\right)+P\left(\left|\hat{\tau}_{n}\right| \leq N \ell_{n}, \Delta_{n} \geq-\epsilon / \ell_{n}^{5 / 2}\right) \\
&+P\left(2 \ell_{n}^{5 / 2} \sup _{|s| \leq \ell_{n} N}\left|\hat{Z}_{n}^{x}(s)-\tilde{Z}_{n}^{x, *}(s)\right| \geq \epsilon\right) .
\end{aligned}
$$

Thus, by Lemmas 5.4 and 5.5 and Proposition 5.2, (5.11) holds.

5.4. Proof of Theorem 3.1. In this subsection we present the proof of Theorem 3.1. For $c>0$, define $\tau(c)=\operatorname{argmin}_{s}\left\{W(s)+c s^{2}\right\}$ and $\tau:=\tau(1)$. Noting that $c^{1 / 3}\left(W(s)+c s^{2}\right)$ and $W\left(c^{2 / 3} s\right)+\left(c^{2 / 3} s\right)^{2}$ have identical distributions, it then follows from $\tau(c)=\operatorname{argmin}_{s}\left\{c^{1 / 3}\left(W(s)+c s^{2}\right)\right\}$ that $\tau(c) \stackrel{d}{=} c^{-2 / 3} \tau$. Define

$$
\left\{\begin{aligned}
\hat{Y}_{n}^{x}(s)= & W_{n}(s)+\frac{f_{0}\left(t_{0}\right) g\left(t_{0}\right)}{2 \sigma_{U}} p_{n}^{2}\left(n^{-1 / 3} s\right)+\frac{g\left(t_{0}\right)}{\sigma_{U}} x \ell_{n} p_{n}\left(n^{-1 / 3} s\right), \\
& n^{-1 / 3} s \in\left(-G\left(t_{0}+\chi_{n}\right), 1-G\left(t_{0}+\chi_{n}\right)\right), \\
Y_{n}^{x}(s)= & W_{n}(s)+\frac{f_{0}\left(t_{0}\right)}{2 \sigma_{U} g\left(t_{0}\right)} s^{2}+\frac{1}{\sigma_{U}} x \ell_{n} s, \quad s \in \mathbb{R},
\end{aligned}\right.
$$

where $p_{n}(s)$ is the inverse function of $q_{n}(u):=G\left(t_{0}+\chi_{n}+n^{-1 / 3} u\right)-G\left(t_{0}+\chi_{n}\right)$. Set $\hat{T}_{n}^{x}=\operatorname{argmin}_{s} \hat{Y}_{n}^{x}(s)$ and $T_{n}^{x}=\operatorname{argmin}_{s} Y_{n}^{x}(s)$. Then $\hat{T}_{n}^{x}=n^{1 / 3} q_{n}\left(\hat{\tau}_{n}^{x}\right)$, and

$$
\lim _{n \rightarrow \infty} \ell_{n}^{3} \sup _{|s| \leq N \ell_{n}}\left|n^{1 / 3} q_{n}(s)-g\left(t_{0}\right) s\right|=0 .
$$

When $s \in\left[-\epsilon_{0} n^{1 / 3}, \epsilon_{0} n^{1 / 3}\right]$, we can write

$$
p_{n}\left(n^{-1 / 3} s\right)=\int_{0}^{n^{-1 / 3} s} \frac{n^{1 / 3} d u}{g\left(t_{0}+\chi_{n}+n^{-1 / 3} u\right)}=\int_{0}^{n^{-2 / 3} s} \frac{n^{2 / 3} d v}{g\left(t_{0}+\chi_{n}+v\right)} .
$$


Thus,

$$
\begin{aligned}
& \sup _{|s| \leq N \ell_{n}}\left|p_{n}\left(n^{-1 / 3} s\right)-\frac{s}{g\left(t_{0}+\chi_{n}\right)}\right| \\
& \quad \leq \int_{0}^{n^{-2 / 3} N \ell_{n}} \frac{n^{2 / 3}\left|g\left(t_{0}+\chi_{n}+v\right)-g\left(t_{0}+\chi_{n}\right)\right| d v}{g\left(t_{0}+\chi_{n}+v\right) g\left(t_{0}+\chi_{n}\right)}
\end{aligned}
$$

which implies that when $\ell_{n}=1, \lim _{n \rightarrow \infty} \sup _{|s| \leq N \ell_{n}}\left|p_{n}\left(n^{-1 / 3} s\right)-\frac{s}{g\left(t_{0}\right)}\right|=0$, and that when Assumptions (A1) and (A2) hold,

$$
\lim _{n \rightarrow \infty} \ell_{n}^{3.5} \sup _{|s| \leq N \ell_{n}}\left|p_{n}\left(n^{-1 / 3} s\right)-\frac{s}{g\left(t_{0}\right)}\right|=0
$$

Therefore, if Assumption (A1) holds and $\ell_{n}=1$ for all $n \geq 1$, or Assumption (A2) also holds, then for each $\rho>0$ and any $\delta>0$,

(5.20) $\quad \limsup _{N \rightarrow \infty} \limsup _{n \rightarrow \infty} \frac{1}{\ell_{n}^{3}} \log \sup _{|x| \in[0, \rho]} P\left(\ell_{n}^{5 / 2} \sup _{|s| \leq \ell_{n} N}\left|\hat{Y}_{n}^{x}(s)-Y_{n}^{x}(s)\right| \geq \delta\right)=-\infty$.

By the same arguments as used in the proofs of Lemma 5.4 and Proposition 5.3, the following result holds.

LEMMA 5.6. (i) For each $\rho>0$,

$$
\underset{N \rightarrow \infty}{\limsup } \limsup _{n \rightarrow \infty} \frac{1}{\ell_{n}^{3}} \log \sup _{|x| \in[0, \rho]} P\left(\max \left\{\left|\hat{T}_{n}^{x}\right|,\left|T_{n}^{x}\right|\right\} \geq \ell_{n} N\right)=-\infty .
$$

(ii) If Assumption (A1) holds and $\ell_{n}=1$ for all $n \geq 1$, or Assumption (A2) also holds, then for each $\rho>0$ and any $\delta>0$,

$$
\limsup _{n \rightarrow \infty} \frac{1}{\ell_{n}^{3}} \log \sup _{|x| \in[0, \rho]} P\left(\left|\hat{T}_{n}^{x}-T_{n}^{x}\right| \geq \delta \ell_{n}^{-2}\right)=-\infty
$$

Write

$$
\begin{aligned}
T_{n}^{x}= & \underset{s}{\operatorname{argmin}}\left\{W_{n}\left(s-\frac{x g\left(t_{0}\right) \ell_{n}}{f_{0}\left(t_{0}\right)}\right)-W_{n}\left(-\frac{x g\left(t_{0}\right) \ell_{n}}{f_{0}\left(t_{0}\right)}\right)+\frac{f_{0}\left(t_{0}\right)}{2 \sigma_{U} g\left(t_{0}\right)} s^{2}\right\} \\
& -\frac{x g\left(t_{0}\right) \ell_{n}}{f_{0}\left(t_{0}\right)} .
\end{aligned}
$$

Thus, $T_{n}^{x}$ and $-\frac{x g\left(t_{0}\right) \ell_{n}}{f_{0}\left(t_{0}\right)}+\left(\frac{f_{0}^{2}\left(t_{0}\right)}{4 F_{0}\left(t_{0}\right)\left(1-F_{0}\left(t_{0}\right)\right) g^{2}\left(t_{0}\right)}\right)^{-1 / 3} \tau$ are identical in distribution. 
Now we are ready to complete the proof of the theorem.

Proof of Theorem 3.1. We only show (3.1). Take $\ell_{n}=b_{n}$. Then for each $\rho>0$, by (5.18), Lemma 5.6 and (2.1), we have

$$
\begin{aligned}
& \lim _{\delta \downarrow 0} \liminf _{n \rightarrow \infty} \inf _{x \in[0, \rho]} \frac{1}{1-F_{\tau}\left(b_{n}\left(\sigma_{F}^{2}\left(t_{0}\right)\right)^{-1 / 3} x\right)} P\left(\hat{\tau}_{n}^{-x}<-b_{n}^{-2} \delta\right) \\
&=\lim _{\delta \downarrow 0} \liminf _{n \rightarrow \infty} \inf _{x \in[0, \rho]} \frac{1}{1-F_{\tau}\left(b_{n}\left(\sigma_{F}^{2}\left(t_{0}\right)\right)^{-1 / 3} x\right)} \\
& \times P\left(\hat{T}_{n}^{-x}<n^{1 / 3} q_{n}\left(-b_{n}^{-2} \delta\right)\right) \\
&= \lim _{\delta \downarrow 0} \liminf _{n \rightarrow \infty} \inf _{x \in[0, \rho]} \frac{1}{1-F_{\tau}\left(b_{n}\left(\sigma_{F}^{2}\left(t_{0}\right)\right)^{-1 / 3} x\right)} P\left(T_{n}^{-x}<-b_{n}^{-2} \delta\right) \\
&= \lim _{\delta \downarrow 0} \liminf _{n \rightarrow \infty} \inf _{x \in[0, \rho]} \frac{1}{1-F_{\tau}\left(b_{n}\left(\sigma_{F}^{2}\left(t_{0}\right)\right)^{-1 / 3} x\right)} \\
& \times P\left(\tau<-b_{n}^{-2} \delta-b_{n}\left(\sigma_{F}^{2}\left(t_{0}\right)\right)^{-1 / 3} x\right) \\
&= 1
\end{aligned}
$$

and

$$
\limsup _{n \rightarrow \infty} \sup _{x \in[0, \rho]} \frac{1}{1-F_{\tau}\left(b_{n}\left(\sigma_{F}^{2}\left(t_{0}\right)\right)^{-1 / 3} x\right)} P\left(\hat{\tau}_{n}^{-x} \leq 0\right)=1 .
$$

Thus, by Propositions 5.3 and 5.1, this yields

$$
\lim _{n \rightarrow \infty} \sup _{x \in\left[0, \rho b_{n}\right]}\left|\frac{1}{1-F_{\tau}(x)} P\left(\frac{n^{1 / 3}\left(\hat{F}_{n}\left(t_{0}+\chi_{n}\right)-F_{0}\left(t_{0}+\chi_{n}\right)\right)}{\left(\sigma_{F}^{2}\left(t_{0}\right)\right)^{1 / 3}} \geq x\right)-1\right|=0 .
$$

In particular, we obtain that for any $x>0$,

$$
\lim _{n \rightarrow \infty} \frac{1}{b_{n}^{3}} \log P\left( \pm \frac{1}{b_{n}} \frac{n^{1 / 3}\left(\hat{F}_{n}\left(t_{0}+\chi_{n}\right)-F_{0}\left(t_{0}+\chi_{n}\right)\right)}{\left(\sigma_{F}^{2}\left(t_{0}\right)\right)^{1 / 3}}>x\right)=-\frac{2}{3} x^{3} .
$$

Equation (5.23) is equivalent to the statement that the sequence $\left\{\frac{n^{1 / 3}}{b_{n}}\left(\hat{F}_{n}\left(t_{0}+\chi_{n}\right)-\right.\right.$ $\left.\left.F_{0}\left(t_{0}+\chi_{n}\right)\right), n \geq 1\right\}$ satisfies the MDP in $\mathbb{R}$ with speed $b_{n}^{3}$ and rate function $I(x)$ [cf. Dembo and Zeitouni (1998), page 7].

5.5. Proof of Theorem 3.2. The proof of Theorem 3.2 is similar to that of Theorem 3.1. Take $\ell_{n} \equiv 1$. Then for $x \in \mathbb{R}$, by Propositions 5.1 and 5.3, Lemma 5.6 
and (5.18), we have

$$
\begin{aligned}
\limsup _{n \rightarrow \infty} & P\left(n^{1 / 3}\left(\hat{F}_{n}\left(t_{0}+\chi_{n}\right)-F_{0}\left(t_{0}+\chi_{n}\right)\right) \leq x\right) \\
\quad \leq & \lim _{\delta \downarrow 0} \limsup _{n \rightarrow \infty} P\left(\tau_{n}^{-x}>-\delta\right) \\
\quad= & \lim _{\delta \downarrow 0} P\left(\frac{x g\left(t_{0}\right)}{f_{0}\left(t_{0}\right)}+\left(\frac{f_{0}^{2}\left(t_{0}\right)}{4 F_{0}\left(t_{0}\right)\left(1-F_{0}\left(t_{0}\right)\right) g^{2}\left(t_{0}\right)}\right)^{-1 / 3} \tau>-\delta\right) \\
\quad= & P\left(\left(\frac{4 f_{0}\left(t_{0}\right) F_{0}\left(t_{0}\right)\left(1-F_{0}\left(t_{0}\right)\right)}{g\left(t_{0}\right)}\right)^{1 / 3} \tau \leq x\right)
\end{aligned}
$$

and

$$
\begin{aligned}
\liminf _{n \rightarrow \infty} P\left(n^{1 / 3}\left(\hat{F}_{n}\left(t_{0}+\chi_{n}\right)-F_{0}\left(t_{0}+\chi_{n}\right)\right) \leq x\right) \\
\quad \geq \liminf _{n \rightarrow \infty} P\left(\tau_{n}^{-x} \geq 0\right) \\
\quad=P\left(\left(\frac{4 f_{0}\left(t_{0}\right) F_{0}\left(t_{0}\right)\left(1-F_{0}\left(t_{0}\right)\right)}{g\left(t_{0}\right)}\right)^{1 / 3} \tau \leq x\right) .
\end{aligned}
$$

Acknowledgements. The authors are very grateful to the Editor, Professor Tailen Hsing, the Associate Editor and the referee for their valuable comments and suggestions that greatly improved the paper.

\section{SUPPLEMENTARY MATERIAL}

Supplement to "Moderate deviations and nonparametric inference for monotone functions" (DOI: 10.1214/17-AOS1583SUPP; .pdf). The supplement [Gao, Xiong and Zhao (2017)] contains all remaining technical proofs omitted from the main text due to space constraints, in which we prove Lemmas 5.4 and 5.5, Proposition 2.2, Theorem 2.3 and its Corollaries 2.1-2.3.

\section{REFERENCES}

Abrevaya, J. and HuAng, J. (2005). On the bootstrap of the maximum score estimator. Econometrica 73 1175-1204. MR2149245

BANERJEE, M. (2007). Likelihood based inference for monotone response models. Ann. Statist. 35 931-956. MR2341693

Banerjee, M. and Wellner, J. A. (2001). Likelihood ratio tests for monotone functions. Ann. Statist. 29 1699-1731. MR1891743

BAnerjee, M. and Wellner, J. A. (2005a). Confidence intervals for current status data. Scand. J. Stat. 32 405-424. MR2204627

BAnerJeE, M. and Wellner, J. A. (2005b). Score statistics for current status data: Comparisons with likelihood ratio and Wald statistics. Int. J. Biostat. 1 Article ID 3. MR2232228

Chang, J., ShaO, Q.-M. and Zhou, W.-X. (2016). Cramér-type moderate deviations for Studentized two-sample $U$-statistics with applications. Ann. Statist. 44 1931-1956. MR3546439 
Dembo, A. and Zeitouni, O. (1998). Large Deviations Techniques and Applications, 2nd ed. Applications of Mathematics (New York) 38. Springer, New York. MR1619036

DE LA PeÑA, V. H., LAI, T. L. and ShaO, Q.-M. (2009). Self-Normalized Processes: Limit Theory and Statistical Applications. Springer, Berlin. MR2488094

DÜMBgen, L., Wellner, J. A. and WolfF, M. (2016). A law of the iterated logarithm for Grenander's estimator. Stochastic Process. Appl. 126 3854-3864. MR3565482

DUROT, C. (2002). Sharp asymptotics for isotonic regression. Probab. Theory Related Fields 122 222-240. MR1894068

DUROT, C. (2007). On the $\mathbb{L}_{p}$-error of monotonicity constrained estimators. Ann. Statist. 351080 1104. MR2341699

Durot, C., Kulikov, V. N. and Lopuhä̈, H. P. (2012). The limit distribution of the $L_{\infty}$-error of Grenander-type estimators. Ann. Statist. 40 1578-1608. MR3015036

GAO, F. (2003). Moderate deviations and large deviations for kernel density estimators. $J$. Theoret. Probab. 16 401-418. MR1982035

GAO, F., XIONG, J. and ZHAO, X. (2018). Supplement to "Moderate deviations and nonparametric inference for monotone functions.” DOI:10.1214/17-AOS1583SUPP.

GAO, F. and ZHAO, X. (2011). Delta method in large deviations and moderate deviations for estimators. Ann. Statist. 39 1211-1240. MR2816352

Giné, E. and Guillou, A. (2001). On consistency of kernel density estimators for randomly censored data: Rates holding uniformly over adaptive intervals. Ann. Inst. Henri Poincaré Probab. Stat. 37 503-522. MR1876841

GRENANDER, U. (1956). On the theory of mortality measurement. II. Skand. Aktuarietidskr. 39 125-153. MR0093415

Groeneboom, P. (1985). Estimating a monotone density. In Proceedings of the Berkeley Conference in Honor of Jerzy Neyman and Jack Kiefer, Vol. II (Berkeley, Calif., 1983) 539-555. Wadsworth, Belmont, CA. MR0822052

Groeneboom, P. (1989). Brownian motion with a parabolic drift and Airy functions. Probab. Theory Related Fields 81 79-109. MR0981568

Groeneboom, P. (1996). Lectures on inverse problems. In Lectures on Probability Theory and Statistics (Saint-Flour, 1994). Lecture Notes in Math. 1648 67-164. Springer, Berlin. MR1600884

Groeneboom, P. (2014). Maximum smoothed likelihood estimators for the interval censoring model. Ann. Statist. 42 2092-2137. MR3262478

Groeneboom, P., Hooghiemstra, G. and Lopuhä̈, H. P. (1999). Asymptotic normality of the $L_{1}$ error of the Grenander estimator. Ann. Statist. 27 1316-1347. MR1740109

Groeneboom, P. and Jongbloed, G. (2014). Nonparametric Estimation Under Shape Constraints: Estimators, Algorithms and Asymptotics. Cambridge Series in Statistical and Probabilistic Mathematics 38. Cambridge Univ. Press, New York. MR3445293

Groeneboom, P. and Jongbloed, G. (2015). Nonparametric confidence intervals for monotone functions. Ann. Statist. 43 2019-2054. MR3375875

Groeneboom, P., Jongbloed, G. and Witte, B. I. (2010). Maximum smoothed likelihood estimation and smoothed maximum likelihood estimation in the current status model. Ann. Statist. 38 352-387. MR2589325

Groeneboom, P. and Wellner, J. A. (1992). Information Bounds and Nonparametric Maximum Likelihood Estimation. DMV Seminar 19. Birkhäuser, Basel. MR1180321

Groeneboom, P. and Wellner, J. A. (2001). Computing Chernoff's distribution. J. Comput. Graph. Statist. 10 388-400. MR1939706

Hooghiemstra, G. and Lopuhä̈, H. P. (1998). An extremal limit theorem for the argmax process of Brownian motion minus a parabolic drift. Extremes $1215-240$. MR1814624

HuANG, J. and WELlNER, J. A. (1995). Estimation of a monotone density or monotone hazard under random censoring. Scand. J. Stat. 22 3-33. MR1334065 
LiU, W. and SHAO, Q.-M. (2010). Cramér-type moderate deviation for the maximum of the periodogram with application to simultaneous tests in gene expression time series. Ann. Statist. 38 1913-1935. MR2662363

LiU, W. and SHAO, Q.-M. (2013). A Carmér moderate deviation theorem for Hotelling's $T^{2}$ statistic with applications to global tests. Ann. Statist. 41 296-322. MR3059419

MAumY, M. (2004). Strong approximations for the compound empirical process. Ann. I.S.U.P. 48 69-83. MR2083781

Petrov, V. V. (1975). Sums of Independent Random Variables. Ergebnisse der Mathematik und ihrer Grenzgebiete 82. Springer, New York. Translated from the Russian by A. A. Brown. MR0388499

Prakasa RaO, B. L. S. (1969). Estimation of a unimodal density. Sankhyā Ser. A 31 23-36. MR0267677

Sun, J. (2006). The Statistical Analysis of Interval-Censored Failure Time Data. Springer, New York. MR2287318

TAlagrand, M. (1996). New concentration inequalities in product spaces. Invent. Math. $126505-$ 563. MR1419006

VAN DER VAART, A. W. and WeLlner, J. A. (1996). Weak Convergence and Empirical Processes: With Applications to Statistics. Springer, New York. MR1385671

F. GAO

SCHOOL OF MATHEMATICS

AND STATISTICS

WUHAN UNIVERSITY

WUHAN 430072

CHINA

E-MAIL: fqgao@whu.edu.cn
J. XIONG

DePartment of Mathematics

UNIVERSITY OF MACAU

MACAU

CHINA

E-MAIL: jiexiong@umac.mo

\author{
X. ZHAO \\ DEPARTMENT OF APPLIED \\ MATHEMATICS \\ The Hong Kong Polytechnic \\ UNIVERSITY \\ HONG KONG \\ CHINA \\ E-MAIL: xingqiu.zhao@polyu.edu.hk
}

